\title{
Nonrelativistic open string and Yang-Mills theory
}

\author{
Jaume Gomis, ${ }^{a}$ Ziqi Yan $^{a, b}$ and Matthew $\mathrm{Yu}^{a}$ \\ ${ }^{a}$ Perimeter Institute for Theoretical Physics, \\ 31 Caroline St N, Waterloo, ON N2L $2 Y 5$, Canada \\ ${ }^{b}$ Nordita, KTH Royal Institute of Technology and Stockholm University, \\ Roslagstullsbacken 23, SE-106 91 Stockholm, Sweden \\ E-mail: jgomis@perimeterinstitute.ca, ziqi.yan@su.se, \\ myu@perimeterinstitute.ca
}

ABSTRACT: The classical and quantum worldsheet theory describing nonrelativistic open string theory in an arbitrary nonrelativistic open and closed string background is constructed. We show that the low energy dynamics of open strings ending on $n$ coincident D-branes in flat spacetime is described by a Galilean invariant $\mathrm{U}(n)$ Yang-Mills theory. We also study nonrelativistic open string excitations with winding number and demonstrate that their dynamics can be encoded into a local gauge theory in one higher dimension. By demanding conformal invariance of the boundary couplings, the nonlinear equations of motion that govern the consistent open string backgrounds coupled to an arbitrary closed background (described by a string Newton-Cartan geometry, Kalb-Ramond, and dilaton field) are derived and shown to emerge from a Galilean invariant Dirac-Born-Infeld type action.

KEywords: Bosonic Strings, Sigma Models, D-branes, Renormalization Group

ARXIV EPRINT: 2007.01886 


\section{Contents}

1 Introduction $\quad 1$

2 Flat spacetime $\quad 3$

2.1 Nonrelativistic open string theory 3

2.2 Bargmann symmetry on D-brane 5

$\begin{array}{lll}2.3 & \text { Open string vertex operators } & 7\end{array}$

2.4 Galilean electrodynamics from nonrelativistic open string theory 8

2.5 Galilean Yang-Mills theory from nonrelativistic open string theory 10

$\begin{array}{ll}2.6 & \text { Winding open string backgrounds } \\ \end{array}$

3 Curved backgrounds $\quad \mathbf{1 5}$

$\begin{array}{lll}3.1 \text { Closed strings on a string Newton-Cartan background } & 15\end{array}$

$\begin{array}{lll}3.2 & \text { Dirichlet sigma model for nonrelativistic open strings } & 17\end{array}$

$\begin{array}{ll}3.3 & \text { Covariant background field method } 20\end{array}$

3.4 Beta-functions for open string couplings 24

$\begin{array}{ll}3.5 & \text { Contribution from the dilaton } \\ \end{array}$

4 Nonrelativistic Dirac-Born-Infeld effective theory 28

4.1 Galilean DBI action from a nonrelativistic limit 28

$\begin{array}{ll}4.2 & \text { Equations of motion } \\ & 29\end{array}$

4.3 Galilean electrodynamics on a Newton-Cartan background 30

5 Conclusions $\quad 32$

\section{Introduction}

Relativistic string theory is governed by the most general classically marginal $2 \mathrm{~d}$ relativistic quantum field theory (QFT) with Poincaré symmetry realized as a global symmetry acting on the worldsheet fields: the nonlinear sigma model [1]. In the presence of a boundary, additional marginal vertex operators supported on the boundary can be turned on, which describe a condensate of open strings ending on D-branes [2]. The coupling constants of the 2d QFT emerge as spacetime fields, and their properties are determined by the structure of the 2d QFT. In particular, relativistic strings propagate on a Lorentzian Riemannian manifold, and in the presence of $n$ D-branes, on a background $\mathrm{U}(n)$ vector bundle. ${ }^{1}$ The celebrated field equations describing the propagation of massless particles of various helicities emerge from relativistic string theory at low energies by demanding quantum consistency

\footnotetext{
${ }^{1}$ Not all string theory backgrounds have a straightforward geometric interpretation (e.g. asymmetric orbifolds). They are nonetheless consistent classical string vacua as long as the $2 \mathrm{~d}$ QFT is a CFT.
} 
of the $2 \mathrm{~d}$ QFT on the string worldsheet, that is, by imposing quantum conformal invariance of the bulk and boundary couplings. These include the Einstein, Rarita-Schwinger, YangMills and Dirac equations. For a single D-brane, conformal invariance leads to a nonlinear theory for the curvature of the $\mathrm{U}(1)$ connection: the Dirac-Born-Infeld (DBI) action [2, 3].

Nonrelativistic string theory in flat spacetime was formulated in [4] as a $2 \mathrm{~d}$ relativistic QFT on the worldsheet with a nonrelativistic global symmetry, known as string NewtonCartan symmetry, acting on the string world sheet fields. ${ }^{2}$ Realizing the string NewtonCartan symmetry requires introducing additional worldsheet fields, which give nonrelativistic string theory some of its most salient features [4]. Nonrelativistic string theory is governed by the most general classically marginal 2d relativistic QFT with string NewtonCartan symmetry acting on the worldsheet fields. The target space geometry induced by the 2d QFT couplings is the string Newton-Cartan geometry [7] (see also [8-12]), ${ }^{3}$ which is to nonrelativistic string theory what Lorentzian Riemannian geometry is to relativistic string theory. The equations of motion that determine the closed string backgrounds in which nonrelativistic strings can consistently propagate were derived in [14]. These are to nonrelativistic string theory what the (super)gravity equations of motion are to relativistic closed string theory.

In this paper, we study nonrelativistic open string theory in open and closed string backgrounds. ${ }^{4}$ We determine the open string background fields in nonrelativistic open string theory by studying the space of open string vertex operators, and derive the backgrounds in which nonrelativistic open string theory can be consistently defined quantum mechanically. Our analysis leads to interesting gauge theories with nonrelativistic symmetry living on the D-branes on which nonrelativistic open strings end.

We derive from a worldsheet analysis a nonlinear $\mathrm{U}(n)$ Yang-Mills action with Galilean symmetry $^{5}$ in flat spacetime,

$$
S_{\mathrm{YM}}=\frac{1}{g_{\mathrm{YM}}^{2}} \int d X^{0} d X^{A^{\prime}} \operatorname{tr}\left(\frac{1}{2} D_{0} N D_{0} N-E_{A^{\prime}} D_{A^{\prime}} N-\frac{1}{4} F_{A^{\prime} B^{\prime}} F_{A^{\prime} B^{\prime}}\right)
$$

where $E_{A^{\prime}}$ and $F_{A^{\prime} B^{\prime}}$ are gauge covariant electric and magnetic fields and $N$ is a scalar in the adjoint representation. The theory for $n=1$ living on a single D-brane is quadratic and reduces to Galilean Electrodynamics [18-20]. The gauge theory (1.1) describes the most general open string background with vanishing winding number to lowest order in the $\alpha^{\prime}$ expansion.

We determine the spacetime effective gauge theory describing open string fields with nontrivial winding number. In spite that winding introduces inherent nonlocalities in string theory, we show that the nonlocality can be tamed by introducing an additional spacetime

\footnotetext{
${ }^{2}$ The nonrelativistic spectrum was first obtained by taking a limit of relativistic string theory in [5] (see also [6]).

${ }^{3}$ For nonrelativistic geometries from null reduction of relativistic string theory see [13] and section 3.1.

${ }^{4}$ Nonrelativistic open string theory corresponds to choosing a Dirichlet boundary condition along the longitudinal spatial direction [15] while a Neumann boundary condition [4] leads to noncommutative open string theory $[16,17]$. See section 2.

${ }^{5}$ More precisely, Bargmann symmetry. See section 2.
} 
dimension on the D-brane, which has the physical interpretation as the coordinate conjugate to winding number. We derive the equations of motion describing the background fields coupling to wound vertex operators and show that the effective action we derive in one higher dimension beautifully reproduces the spectrum and mass shell condition of the corresponding excitations of nonrelativistic open string theory.

Finally, we couple nonrelativistic string theory to an arbitrary closed and open string background. By imposing quantum mechanical conformal invariance on the open string couplings, we find a nonlinear system of equations of motion describing the open string fields. We show that these equations of motion can be derived from a nonlinear local field theory which has Galilean symmetry and which we dub Galilean DBI.

The plan for the rest of the paper is as follows. In the first half of section 2 , we introduce nonrelativistic string theory in flat spacetime and classify the open string vertex operators. In the second half of section 2, we determine the nonrelativistic gauge theories living on D-branes by requiring that the open string vertex operators be conformally invariant at the quantum level. In section 3, we study nonrelativistic string theory in an arbitrary closed and open string background and derive the one-loop beta-functions for the boundary coupling constants in the corresponding Dirichlet sigma model. In section 4, we put forward the Galilean DBI action whose equations of motion reproduce the vanishing beta-functions. In section 5, we conclude our paper.

\section{Flat spacetime}

\subsection{Nonrelativistic open string theory}

The worldsheet action of nonrelativistic string theory with flat target spacetime in conformal gauge is [4]

$$
S_{\text {flat }}=\frac{1}{4 \pi \alpha^{\prime}} \int_{\Sigma} d^{2} z\left(2 \partial_{z} X^{A^{\prime}} \partial_{\bar{z}} X^{A^{\prime}}+\lambda \partial_{\bar{z}} X+\bar{\lambda} \partial_{z} \bar{X}\right)
$$

where

$$
X=X^{0}+X^{1}, \quad \bar{X}=X^{0}-X^{1},
$$

and

$$
z=\sigma+i \tau, \quad \bar{z}=\sigma-i \tau .
$$

It follows that

$$
\partial_{z}=\frac{1}{2}\left(\partial_{\sigma}-i \partial_{\tau}\right), \quad \partial_{\bar{z}}=\frac{1}{2}\left(\partial_{\sigma}+i \partial_{\tau}\right) .
$$

The worldsheet fields are the worldsheet scalars parametrizing the spacetime coordinates $X^{\mu}=\left(X^{A}, X^{A^{\prime}}\right)$, with $A=0,1$ and $A^{\prime}=2, \cdots, d-1$, where $d$ denotes the spacetime dimension, and two additional one-form fields $\lambda$ and $\bar{\lambda}$. The fields $X^{A}$ parametrize longitudinal coordinates and $X^{A^{\prime}}$ transverse coordinates. The Riemann surface $\Sigma$ is parametrized by the Euclidean coordinates $\sigma^{\alpha}=(\tau, \sigma)$. The critical dimension is $d=26$ and $d=10$ for bosonic and supersymmetric nonrelativistic string theory. 
Let us consider open string theory by analyzing the boundary conditions for the worldsheet fields on a surface $\Sigma$ with a boundary $\partial \Sigma$ at $\sigma=0$. Taking variations with respect to the worldsheet fields in (2.1) and requiring the bulk equations of motion,

$$
\begin{aligned}
\partial_{\bar{z}} X=\partial_{z} \bar{X} & =0, \\
\partial_{\bar{z}} \lambda=\partial_{z} \bar{\lambda} & =0, \\
\partial_{z} \partial_{\bar{z}} X^{A^{\prime}} & =0,
\end{aligned}
$$

to hold, we find the following boundary contribution:

$$
\delta S_{\text {flat }}=\frac{1}{2 \pi \alpha^{\prime}} \int_{\partial \Sigma} d \tau\left[\delta X^{A^{\prime}} \partial_{\sigma} X^{A^{\prime}}+\frac{1}{2}(\lambda+\bar{\lambda}) \delta X^{0}+\frac{1}{2}(\lambda-\bar{\lambda}) \delta X^{1}\right] .
$$

We impose a Dirichlet boundary condition in the longitudinal spatial $X^{1}$-direction $[15]^{6}$

$$
\left.X^{1}\right|_{\sigma=0}=X_{0}^{1}
$$

where $X_{0}^{1}$ is constant. This implies $\left.\delta X^{1}\right|_{\sigma=0}=0$. Furthermore, varying with respect to $X^{0}$ gives rise to the following boundary condition for the one-form fields

$$
\lambda+\left.\bar{\lambda}\right|_{\sigma=0}=0 .
$$

The equations of motion of $X$ and $\bar{X}$ imply that $X^{0}$ obeys a Neumann boundary condition

$$
\left.\partial_{\sigma} X^{0}\right|_{\sigma=0}=0
$$

and that ${ }^{7}$

$$
\partial_{\sigma} X^{1}+\left.i \partial_{\tau} X^{0}\right|_{\sigma=0}=0
$$

The transverse coordinates behave the same as in conventional string theory, and henceforth we consider Neumann boundary conditions for the transverse coordinates $X^{A^{\prime}}$

$$
\left.\partial_{\sigma} X^{A^{\prime}}\right|_{\sigma=0}=0
$$

These boundary conditions preserve conformal invariance since (2.8) and (2.10) implies that $\left.\left(\partial_{z} X+\partial_{\bar{z}} \bar{X}\right)\right|_{\sigma=0}=0$, which with $(2.9)$ and $(2.12)$ says that $\left.(T-\bar{T})\right|_{\sigma=0}=0 .{ }^{8}$ These boundary conditions define the open string theory on a $\mathrm{D}(d-2)$-brane that is transverse to the longitudinal spatial $X^{1}$-direction.

\footnotetext{
${ }^{6}$ We can also consider boundary conditions in the presence of a constant longitudinal $B$-field

$$
\frac{1}{4 \pi \alpha^{\prime}} \int_{\Sigma} d^{2} \sigma \epsilon^{\alpha \beta} \partial_{\alpha} X^{A} \partial_{\beta} X^{B} \epsilon_{A B}=-\frac{1}{2 \pi \alpha^{\prime}} \int_{\partial \Sigma} d \tau X^{0} \partial_{\tau} X^{1}
$$

This term is a total derivative and it vanishes when the Dirichlet boundary condition in (2.8) is introduced, but otherwise leads to [4] noncommutative open string theory $[16,17]$ on a spacetime-filling brane.

${ }^{7}$ Equation (2.11) relates the derivatives $\partial_{\sigma} X^{1}$ and $\partial_{\tau} X^{0}$ at the boundary of the worldsheet, and will play an important role in section 2.3 when studying the space of open string vertex operators.

${ }^{8}$ See (2.34) for the formula for the worldsheet stress energy tensor $T$.
} 
The worldsheet theory (2.1) with these boundary conditions defines nonrelativistic open string theory in flat spacetime, which has an open string spectrum with a Galilean invariant dispersion relation. The nonrelativistic open string energy spectrum is [15]

$$
p_{0}=\alpha^{\prime} \frac{p_{A^{\prime}} p_{A^{\prime}}}{2 w R}+\frac{N_{\mathrm{open}}}{2 w R},
$$

where the $X^{1}$-direction is taken to be a circle of radius $R .{ }^{9}$ We denote the winding number along $X^{1}$ as $w$, the open string excitation number as $N_{\text {open }}$, and the transverse spacetime momentum as $p_{A^{\prime}}$. Since the spectrum (2.13) is singular when $w=0$, all asymptotic states must necessarily have nonzero winding (unless $p_{A^{\prime}}=0$ ). However, off-shell states in the zero winding sector play the role of intermediate states that mediate an instantaneous electromagnetic force between winding strings, akin to closed string states with zero winding mediating instantaneous gravitational forces.

One can nevertheless deform the worldsheet theory (2.1) with open string vertex operators with $w=0$. This changes the background fields in which nonrelativitsic open strings propagate. In this paper, we determine the open string backgrounds on which nonrelativistic string theory can be consistently defined. In the following, we mostly focus on the zero winding sector. We will discuss nonzero windings in section 2.6.

\subsection{Bargmann symmetry on D-brane}

Now, we discuss the global symmetries of the nonrelativistic action in flat space (2.1). When the surface $\Sigma$ has no boundary, the action (2.1) is invariant under the following infinitesimal global symmetry transformations:

$$
\begin{aligned}
\delta X^{A^{\prime}} & =g^{A^{\prime}}(X)+\bar{g}^{A^{\prime}}(\bar{X})-\Lambda^{A^{\prime}}{ }_{B^{\prime}} X^{B^{\prime}}, \\
\delta X & =f(X), \quad \delta \lambda=-\lambda \partial_{X} f(X)-2 \partial_{X} g_{A^{\prime}}(X) \partial X^{A^{\prime}}, \\
\delta \bar{X} & =\bar{f}(\bar{X}), \quad \delta \bar{\lambda}=-\bar{\lambda} \partial_{\bar{X}} \bar{f}(\bar{X})-2 \partial_{\bar{X}} \bar{g}_{A^{\prime}}(\bar{X}) \bar{\partial} X^{A^{\prime}} .
\end{aligned}
$$

These transformations form the so-called "extended Galilean symmetry algebra", which contains two copies of the Witt algebra [21]. This symmetry algebra reduces to the string Newton-Cartan algebra when coupling nonrelativistic string theory to general background fields (see section 3.1).

Let us now determine the symmetries preserved by a $\mathrm{D}(d-2)$-brane. This requires finding the transformations (2.14) that preserve the boundary conditions $(2.8)-(2.12)$ on the boundary of the worldsheet. First, these conditions impose that

$$
\partial_{X} g^{A^{\prime}}(X)=\partial_{\bar{X}} \bar{g}^{A^{\prime}}(\bar{X}), \quad \partial_{X} f(X)=\partial_{\bar{X}} \bar{f}(\bar{X})
$$

for arbitrary $X$ and $\bar{X}$. This implies that $g^{A^{\prime}}, \bar{g}^{A^{\prime}}, f$ and $\bar{f}$ are functions at most linear in their arguments. Moreover, imposing that the transformation preserves the Dirichlet

\footnotetext{
${ }^{9}$ We can also consider open strings stretched between D-branes separated by a distance $L$ in the $X^{1}$ direction. Then $w R$ in $(2.13)$ must be replaced with $L /(2 \pi)$.
} 
boundary condition along $X^{1}$ correlates the constant part of $f$ with that of $\bar{f}$. Thus, the infinitesimal symmetry transformations of nonrelativistic open string theory are:

$$
\begin{array}{rlrl}
\delta X^{A^{\prime}} & =\Xi^{A^{\prime}}+\Lambda^{A^{\prime}} X^{0}-\Lambda^{A^{\prime}}{ }_{B^{\prime}} X^{B^{\prime}}, \\
\delta X^{0} & =\Xi^{0}+\Theta X^{0}, & \delta \lambda=-\Theta \lambda-2 \Lambda_{A^{\prime}} \partial_{z} X^{A^{\prime}}, \\
\delta X^{1} & =\Theta X^{1}, & \delta \bar{\lambda}=-\Theta \bar{\lambda}-2 \Lambda_{A^{\prime}} \partial_{\bar{z}} X^{A^{\prime}} .
\end{array}
$$

Evaluated on the worldsheet boundary, we have

$$
\delta \lambda=-\delta \bar{\lambda}=-\Theta \lambda+i \Lambda_{A^{\prime}} \partial_{\tau} X^{A^{\prime}} .
$$

The conserved charges associated to the different transformations are:

$$
\begin{aligned}
\text { time translation } & \Xi^{0}: & H & =\int d \sigma \pi_{0}, \\
\text { transverse translation } & \Xi^{A^{\prime}}: & P_{A^{\prime}} & =\int d \sigma \pi_{A^{\prime}}, \\
\text { Galilean boost } & \Lambda^{A^{\prime}}: & G_{A^{\prime}} & =\int d \sigma\left(X^{0} \pi_{A^{\prime}}-\frac{i}{2 \pi \alpha^{\prime}} X^{A^{\prime}} \partial_{\sigma} X^{1}\right), \\
\text { transverse rotation } & \Lambda^{A^{\prime}}{ }_{B^{\prime}}: & J_{A^{\prime} B^{\prime}} & =\int d \sigma\left(-X^{A^{\prime}} \pi_{B^{\prime}}+X^{B^{\prime}} \pi_{A^{\prime}}\right), \\
\text { longitudinal dilatation } & \Theta: & D & =\int d \sigma X^{A} \pi_{A},
\end{aligned}
$$

where we defined the conjugate momentum for $X^{0}, X^{1}$ and $X^{A^{\prime}}$ as

$$
\pi_{0}=\frac{i}{4 \pi \alpha^{\prime}}(\lambda-\bar{\lambda}), \quad \pi_{1}=\frac{i}{4 \pi \alpha^{\prime}}(\lambda+\bar{\lambda}), \quad \pi_{A^{\prime}}=\frac{1}{2 \pi \alpha^{\prime}} \partial_{\tau} X^{A^{\prime}} .
$$

Using the equal- $\tau$ Poisson brackets,

$$
\left[X^{A}\left(\sigma_{1}\right), \pi_{B}\left(\sigma_{2}\right)\right]=\delta_{B}^{A} \delta\left(\sigma_{1}-\sigma_{2}\right), \quad\left[X^{A^{\prime}}\left(\sigma_{1}\right), \pi_{B^{\prime}}\left(\sigma_{2}\right)\right]=\delta_{B^{\prime}}^{A^{\prime}} \delta\left(\sigma_{1}-\sigma_{2}\right),
$$

we find that the generators $H, P_{A^{\prime}}, G_{A^{\prime}}$ and $J_{A^{\prime} B^{\prime}}$ satisfy the Bargmann algebra,

$$
\begin{array}{rlrl}
{\left[H, G_{A^{\prime}}\right]} & =-P_{A^{\prime}}, & {\left[P_{A^{\prime}}, J_{B^{\prime} C^{\prime}}\right]=\delta_{A^{\prime} B^{\prime}} P_{C^{\prime}}-\delta_{A^{\prime} C^{\prime}} P_{B^{\prime}},} \\
{\left[P_{A^{\prime}}, G_{B^{\prime}}\right]} & =\delta_{A^{\prime} B^{\prime}} Z, & {\left[G_{A^{\prime}}, J_{B^{\prime} C^{\prime}}\right]=\delta_{A^{\prime} B^{\prime}} G_{C^{\prime}}-\delta_{A^{\prime} C^{\prime}} G_{B^{\prime}},} \\
{\left[J_{A^{\prime} B^{\prime}}, J_{C^{\prime} D^{\prime}}\right]} & =\delta_{B^{\prime} C^{\prime}} J_{A^{\prime} D^{\prime}}-\delta_{A^{\prime} C^{\prime}} J_{B^{\prime} D^{\prime}}+\delta_{A^{\prime} D^{\prime}} J_{B^{\prime} C^{\prime}}-\delta_{B^{\prime} D^{\prime}} J_{A^{\prime} C^{\prime}},
\end{array}
$$

where

$$
Z=\frac{i}{2 \pi \alpha^{\prime}} \int d \sigma \partial_{\sigma} X^{1}
$$

is the central charge in the Bargmann algebra. Note that $Z$ measures the winding number of strings along $X^{1}$. The appearance of the Bargmann algebra here is expected, as the open string theory defines a QFT living on the D-brane, which describes dynamics of nonrelativistic gauge fields that we will elaborate on in the rest of the section.

This symmetry algebra is further extended by the longitudinal dilatation generator in $(2.18 \mathrm{e})$, which satisfies the following Lie brackets:

$$
[D, H]=H, \quad\left[D, G_{A^{\prime}}\right]=-G_{A^{\prime}}, \quad[D, Z]=-Z .
$$


Note that the compactified circle in $X^{1}$ is rescaled under the dilatation transformation. This rescaling can be compensated when a dilaton is included. Consequently, as we will see later at the end of section 2.4, the dilatation is only a symmetry of the spacetime equations of motion instead of the spacetime action, unless a dilaton background field is present. Also note that the dilatation generator in (2.18e) gives rise to the dilaton background in string theory when the algebra is gauged. ${ }^{10}$

\subsection{Open string vertex operators}

Our next goal is to consider the most general deformation of nonrelativistic open string theory in flat spacetime by perturbing around the free action (2.1) with open string vertex operators. We consider here vertex operators with zero winding along $X^{1}$. Turning on these vertex operators changes the background fields in which nonrelativistic open strings propagate.

In order to classify the open string vertex operators that can be added to the sigma model action $(2.1)$, we first consider all possible $(1,0)$ and $(0,1)$ forms in the bulk,

$$
\lambda, \quad \partial_{z} X, \quad \partial_{z} X^{A^{\prime}} ; \quad \bar{\lambda}, \quad \partial_{\bar{z}} \bar{X}, \quad \partial_{\bar{z}} X^{A^{\prime}} .
$$

In section 2.1, we derived the boundary conditions in nonrelativistic open string theory with a flat target space (and with zero open string background fields), which we collect as follows:

$$
\begin{aligned}
\text { Dirichlet: } & \left.\partial_{\tau} X^{1}\right|_{\sigma=0}=0 \\
\text { Neumann: } & \left.\partial_{\sigma} X^{0}\right|_{\sigma=0}=\left.\partial_{\sigma} X^{A^{\prime}}\right|_{\sigma=0}=0,
\end{aligned}
$$

and

$$
\lambda+\left.\bar{\lambda}\right|_{\sigma=0}=\partial_{\sigma} X^{1}+\left.i \partial_{\tau} X^{0}\right|_{\sigma=0}=0 .
$$

Therefore, the independent one-forms in (2.24) are

$$
\partial_{\tau} X^{0}, \quad \partial_{\tau} X^{A^{\prime}}, \quad \lambda .
$$

The most general deformation created by an open string vertex operator with zero winding is

$$
\mathcal{V}=\int_{\partial \Sigma} d \tau\left[: N \lambda:+i\left(: A_{0} \partial_{\tau} X^{0}:+: A_{A^{\prime}} \partial_{\tau} X^{A^{\prime}}:\right)\right] .
$$

The worldsheet couplings $N, A_{0}$ and $A_{A^{\prime}}$ are functions of $X^{0}$ and $X^{A^{\prime}}$ and have a spacetime interpretation as background open string fields on the $\mathrm{D}(d-2)$-brane worldvolume. Here, $A_{0}$ and $A_{A^{\prime}}$ are the components of a $\mathrm{U}(1)$ gauge field. The vertex operator (2.28) is invariant under the $\mathrm{U}(1)$ gauge transformation

$$
\delta_{\epsilon} A_{0}=\partial_{0} \epsilon, \quad \delta_{\epsilon} A_{A^{\prime}}=\partial_{A^{\prime}} \epsilon .
$$

\footnotetext{
${ }^{10}$ See [12] for relevant discussion of the analogue of this dilatation generator in string Newton-Cartan algebra.
} 
The coupling $N=N\left(X^{0}, X^{A^{\prime}}\right)$, which is invariant under the $\mathrm{U}(1)$ gauge symmetry, has a geometrical interpretation as the Nambu-Goldstone boson that perturbs around the solitonic $\mathrm{D}(d-2)$-brane, which breaks the translational symmetry along the $X^{1}$ direction.

The vertex operator (2.28) is invariant under the global spacetime symmetry algebra we derived in the previous section when supplementing the worldsheet transformations in (2.16) with the following infinitesimal transformations of the background fields:

$$
\begin{aligned}
\delta N & =-\xi^{0} \partial_{0} N-\xi^{A^{\prime}} \partial_{A^{\prime}} N+\Theta N \\
\delta A_{0} & =-\xi^{0} \partial_{0} A_{0}-\xi^{A^{\prime}} \partial_{A^{\prime}} A_{0}-A_{0} \partial_{0} \xi^{0}-A_{A^{\prime}} \partial_{0} \xi^{A^{\prime}}, \\
\delta A_{A^{\prime}} & =-\xi^{0} \partial_{0} A_{A^{\prime}}-\xi^{B^{\prime}} \partial_{B^{\prime}} A_{A^{\prime}}-A_{0} \partial_{A^{\prime}} \xi^{0}-A_{B^{\prime}} \partial_{A^{\prime}} \xi^{B^{\prime}}-\Lambda_{A^{\prime}} N,
\end{aligned}
$$

where

$$
\xi^{0} \equiv \Xi^{0}+\Theta X^{0}, \quad \xi^{A^{\prime}} \equiv \Xi^{A^{\prime}}+\Lambda^{A^{\prime}} X^{0}-\Lambda^{A^{\prime}}{ }_{B^{\prime}} X^{B^{\prime}} .
$$

In particular, the transformations under the Galilean boost symmetry are

$$
\begin{aligned}
\delta_{G} N & =-\Lambda^{A^{\prime}} X^{0} \partial_{A^{\prime}} N, \\
\delta_{G} A_{0} & =-\Lambda^{A^{\prime}} X^{0} \partial_{A^{\prime}} A_{0}-\Lambda^{A^{\prime}} A_{A^{\prime}}, \\
\delta_{G} A_{A^{\prime}} & =-\Lambda^{B^{\prime}} X^{0} \partial_{B^{\prime}} A_{A^{\prime}}-\Lambda_{A^{\prime}} N .
\end{aligned}
$$

These transformations realize on the background fields $\left(A_{0}, A_{A^{\prime}}, N\right)$ the Bargmann algebra (with the additional dilatation symmetry) that is preserved by the D-brane. The expression in (2.28) is the most general open string vertex operator with zero winding respecting these symmetries.

Our goal is to determine the open string backgrounds in which nonrelativistic open strings in flat spacetime can propagate on.

\subsection{Galilean electrodynamics from nonrelativistic open string theory}

Requiring that the open string vertex operator in (2.28) is BRST invariant imposes constraints on the couplings $\left(A_{0}, A_{A^{\prime}}, N\right)$. These conditions are the linearized spacetime equations of motion that the background fields $\left(A_{0}, A_{A^{\prime}}, N\right)$ must satisfy for a self-consistent nonrelativistic open string theory.

The (holomorphic) BRST charge of nonrelativistic string theory (2.1) is

$$
Q=\int \frac{d z}{2 \pi i}\left[: c T^{m}(z):+: b c \partial_{z} c:+\frac{3}{2}: \partial_{z}^{2} c:\right],
$$

where the matter stress energy tensor is

$$
T^{m}(z)=-\frac{1}{2 \alpha^{\prime}}\left(2 \partial_{z} X^{A^{\prime}} \partial_{z} X^{A^{\prime}}+\lambda \partial_{z} X\right) .
$$

Our goal is to calculate the BRST transformation of the most general open string vertex operator (2.28).

Calculating the BRST transformation requires computing operator product expansions (OPEs). Using the Neumann boundary condition (2.12) for $X^{A^{\prime}}$, we find

$$
X^{A^{\prime}}(z, \bar{z}) X^{B^{\prime}}\left(z^{\prime}, \bar{z}^{\prime}\right) \sim-\frac{1}{2} \alpha^{\prime} \delta^{A^{\prime} B^{\prime}}\left(\ln \left|z-z^{\prime}\right|^{2}+\ln \left|z+\bar{z}^{\prime}\right|^{2}\right) .
$$


Moreover, the OPEs for the holomorphic fields $\lambda=\lambda(z)$ and $X=X(z)$, and the antiholomorphic fields $\bar{\lambda}(\bar{z})$ and $\bar{X}(\bar{z})$ are

$$
\lambda(z) X\left(z^{\prime}\right) \sim-\frac{2 \alpha^{\prime}}{z-z^{\prime}}, \quad \bar{\lambda}(\bar{z}) \bar{X}\left(\bar{z}^{\prime}\right) \sim-\frac{2 \alpha^{\prime}}{\bar{z}-\bar{z}^{\prime}} .
$$

We decompose the worldsheet field $X^{\mu}$ into its left- and right-moving parts as

$$
X^{\mu}(z, \bar{z})=X_{L}^{\mu}(z)+X_{R}^{\mu}(\bar{z})
$$

In doing so, the (anti-)holomorphic equations of motion $\bar{\partial} X=\partial \bar{X}=0$ implies

$$
X(z)=2 X_{L}^{0}(z)=2 X_{L}^{1}(z), \quad \bar{X}(\bar{z})=2 X_{R}^{0}(\bar{z})=-2 X_{R}^{1}(\bar{z}) .
$$

In what follows, we focus on the left-moving modes $X_{L}^{A^{\prime}}(z), X_{L}^{0}(z)$, and $\lambda(z)$, with

$$
X_{L}^{A^{\prime}}(z) X_{L}^{B^{\prime}}\left(z^{\prime}\right) \sim-\frac{1}{2} \alpha^{\prime} \delta^{A^{\prime} B^{\prime}} \ln \left(z-z^{\prime}\right), \quad \lambda(z) X_{L}^{0}\left(z^{\prime}\right) \sim-\frac{\alpha^{\prime}}{z-z^{\prime}} .
$$

In terms of the holomorphic fields and the holomorphic coordinate $z$, we write the open string vertex operator $\mathcal{V}$ in $(2.28)$ as

$$
\mathcal{V}=\int_{\partial \Sigma} d z V_{L}(z)-\int_{\partial \Sigma} d \bar{z} V_{R}(\bar{z})
$$

For open strings, the holomorphic and anti-holomorphic parts are related to each other by the boundary conditions, so it is sufficient to focus on either one of them. In what follows, we will study the holomorphic part,

$$
\mathcal{V}_{L}=\int_{\partial \Sigma} d z V_{L}(z)
$$

with

$$
V_{L}(z)=\frac{1}{2}: N \lambda(z):-: A_{0} \partial_{z} X_{L}^{0}(z):-: A_{A^{\prime}} \partial_{z} X_{L}^{A^{\prime}}(z): .
$$

It then follows that the BRST transformation of $V_{L}$ is

$$
\left[Q, V_{L}(z)\right]=: \partial_{z} W_{L}(z):-\frac{\alpha^{\prime}}{4}: \partial_{z} c(z) \mathcal{E}(z):
$$

where

$$
\begin{aligned}
W_{L}(z) & =c(z) V_{L}(z)+\frac{\alpha^{\prime}}{4} \partial_{z} c\left(\partial^{A^{\prime}} A_{A^{\prime}}-\partial_{0} N\right), \\
\mathcal{E}(z) & =\frac{1}{2} \partial^{A^{\prime}} \partial_{A^{\prime}} N \lambda-\left(\partial_{0}^{2} N-\partial^{A^{\prime}} E_{A^{\prime}}\right) \partial X^{0}-\left(\partial_{0} \partial_{A^{\prime}} N+\partial^{B^{\prime}} F_{B^{\prime} A^{\prime}}\right) \partial X^{A^{\prime}},
\end{aligned}
$$

and we have introduced the gauge invariant electric and magnetic fields,

$$
E_{A^{\prime}}=\partial_{0} A_{A^{\prime}}-\partial_{A^{\prime}} A_{0}, \quad F_{A^{\prime} B^{\prime}}=\partial_{A^{\prime}} A_{B^{\prime}}-\partial_{B^{\prime}} A_{A^{\prime}} .
$$


Requiring that $\mathcal{V}_{L}$ in (2.41) is BRST invariant implies that $\left[Q, V_{L}(z)\right]$ must be a total derivative, which requires that $\mathcal{E}=0$. This sets the couplings in front of the operators $\lambda$, $\partial X^{0}$ and $\partial X^{A^{\prime}}$ in $(2.44 \mathrm{~b})$ to zero separately,

$$
\begin{aligned}
\partial^{A^{\prime}} \partial_{A^{\prime}} N & =0, \\
\partial_{0}^{2} N-\partial^{A^{\prime}} E_{A^{\prime}} & =0, \\
\partial_{A^{\prime}} \partial_{0} N+\partial^{B^{\prime}} F_{B^{\prime} A^{\prime}} & =0 .
\end{aligned}
$$

Therefore, when the background fields $\left(A_{0}, A_{A^{\prime}}, N\right)$ satisfy the linearized equations of motion (2.46), nonrelativistic open string theory can be consistently defined.

The linearized background field equations in (2.46) arise from varying the following action with respect to $N, A_{0}$ and $A_{A^{\prime}}$,

$$
S=\frac{1}{g^{2}} \int d X^{0} d X^{A^{\prime}}\left(\frac{1}{2} \partial_{0} N \partial_{0} N-E_{A^{\prime}} \partial_{A^{\prime}} N-\frac{1}{4} F_{A^{\prime} B^{\prime}} F_{A^{\prime} B^{\prime}}\right),
$$

where $g^{2}=e^{\Phi_{0}}$, with $\Phi_{0}$ the dilaton expectation value. This theory is known as Galilean Electrodynamics in the literature, which was historically discovered by considering a null reduction of Maxwell theory in [18], and later reproduced as a nonrelativistic limit of Maxwell theory with a free massless scalar in $[19,20] .{ }^{11}$ The action $(2.47)$ is invariant under the Bargmann symmetry, and if we appropriately shift the dilaton, also under the dilatation symmetry $(2.18 \mathrm{e})$, that is, dilatation transformations are a symmetry of the equations of motion but not of the action (2.47).

As mentioned earlier, in the zero winding sector, there are no propagating degrees of freedom. Nevertheless, strings with no winding can appear as intermediate states that mediate instantaneous electromagnetic forces between winding strings. The fact that open strings with no winding do not have propagating degrees of freedom is realized in the absence of propagating modes in the Galilean Electrodynamics action (2.47).

In section 3, we generalize the Galilean Electrodynamics result in (2.46) to its DBI analogue in arbitrary open and closed background fields.

\subsection{Galilean Yang-Mills theory from nonrelativistic open string theory}

Our next goal is to derive the low energy effective action that determines the consistent open string backgrounds on a stack of $n$ coincident $\mathrm{D}(d-2)$-branes. This requires introducing

\footnotetext{
${ }^{11}$ In [20], a finite Galilean boost transformation was introduced, under which (2.47) is invariant. In the worldsheet formalism we are working with, if one takes the boost transformation $X^{A} \rightarrow X^{A}$ and $X^{A^{\prime}} \rightarrow X^{A^{\prime}}+\Lambda^{A^{\prime}} X^{0}$ in (2.16) to be a finite transformation, then, by requiring the nonrelativistic string action (2.1) to be invariant, the boost transformation of the one-form fields $\lambda$ and $\bar{\lambda}$ gain a term quadratic in $\Lambda_{A^{\prime}}$,

$$
\lambda \rightarrow \lambda-\Lambda_{A^{\prime}} \partial X^{A^{\prime}}-\frac{1}{2} \Lambda_{A^{\prime}} \Lambda^{A^{\prime}} \partial X^{0}, \quad \bar{\lambda} \rightarrow \bar{\lambda}-\Lambda_{A^{\prime}} \bar{\partial} X^{A^{\prime}}-\frac{1}{2} \Lambda_{A^{\prime}} \Lambda^{A^{\prime}} \bar{\partial} X^{0} .
$$

Requiring the open string vertex operator (2.28) to be invariant under these transformations gives

$$
\begin{aligned}
N^{\prime}\left(X^{\prime}\right)=N(X), \quad A_{0}^{\prime}\left(X^{\prime}\right) & =A_{0}(X)-\Lambda^{A^{\prime}} A_{A^{\prime}}(X)+\frac{1}{2} \Lambda_{A^{\prime}} \Lambda^{A^{\prime}} N(X), \\
A_{A^{\prime}}^{\prime}\left(X^{\prime}\right) & =A_{A^{\prime}}(X)-\Lambda^{A^{\prime}} N,
\end{aligned}
$$

which derives the finite boost transformations given in [20]. 
$\mathrm{U}(n)$ Chan-Paton bundles. The most general open string vertex operator with vanishing winding number is described by the Wilson line like insertion

$$
W=\operatorname{tr} \mathcal{P} \exp \left\{\int_{\partial \Sigma} d \tau\left[: N \lambda:+i\left(: A_{0} \partial_{\tau} X^{0}:+: A_{A^{\prime}} \partial_{\tau} X^{A^{\prime}}:\right)\right]\right\},
$$

where the path-ordering operator $\mathcal{P}$ orders terms such that higher values of $\tau$ stand to the left. We defined the adjoint fields

$$
A_{0}=A_{0}^{m} T^{m}, \quad A_{A^{\prime}}=A_{A^{\prime}}^{m} T^{m}, \quad N=N^{m} T^{m},
$$

where $T^{m}$ are generators in the fundamental representation of $\mathrm{U}(n)$. There are $n^{2}$ generators $T^{m}$, each of which is a hermitian $n \times n$ matrix. The fields in (2.51) transform under $\mathrm{U}(n)$ gauge transformations as follows:

$$
\begin{aligned}
A_{0} & \rightarrow U A_{0} U^{\dagger}+i U\left(\partial_{0} U^{\dagger}\right), \\
A_{A^{\prime}} & \rightarrow U A_{A^{\prime}} U^{\dagger}+i U\left(\partial_{A^{\prime}} U^{\dagger}\right), \\
N & \rightarrow U N U^{\dagger},
\end{aligned}
$$

where

$$
U\left(X_{0}, X_{A^{\prime}}\right)=\exp \left[i \alpha^{m}\left(X_{0}, X_{A^{\prime}}\right) T^{m}\right] .
$$

These leave the vertex operator (2.50) invariant. $N$ is therefore a field in the adjoint representation of $\mathrm{U}(n)$. The gauge-covariant field strengths are

$$
\begin{aligned}
E_{A^{\prime}} & =\partial_{0} A_{A^{\prime}}-\partial_{A^{\prime}} A_{0}-i\left[A_{0}, A_{A^{\prime}}\right], \\
F_{A^{\prime} B^{\prime}} & =\partial_{A^{\prime}} A_{B^{\prime}}-\partial_{B^{\prime}} A_{A^{\prime}}-i\left[A_{A^{\prime}}, A_{B^{\prime}}\right] .
\end{aligned}
$$

We define the covariant derivatives $D_{0}$ and $D_{A^{\prime}}$, which act on the adjoint scalar $N$ as

$$
D_{0} N=\partial_{0} N-i\left[A_{0}, N\right], \quad D_{A^{\prime}} N=\partial_{A^{\prime}} N-i\left[A_{A^{\prime}}, N\right] .
$$

We would like to derive the Yang-Mills analogue of the Galilean Electrodynamics equations of motion in (2.46), which involves keeping track of terms up to the third order in the background fields in (2.50) in the following expansion:

$$
\begin{aligned}
W=\operatorname{tr}[\mathbb{1} & +\int_{-\infty}^{\infty} d \tau V(\tau)+\int_{-\infty}^{\infty} d \tau V(\tau) \int_{-\infty}^{\tau} d \tau^{\prime} V\left(\tau^{\prime}\right) \\
& \left.+\int_{-\infty}^{\infty} d \tau V(\tau) \int_{-\infty}^{\tau} d \tau^{\prime} V\left(\tau^{\prime}\right) \int_{-\infty}^{\tau^{\prime}} d \tau^{\prime \prime} V\left(\tau^{\prime \prime}\right)+\cdots\right]
\end{aligned}
$$

where

$$
V=: N \lambda:+i\left(: A_{0} \partial_{\tau} X^{0}:+: A_{A^{\prime}} \partial_{\tau} X^{A^{\prime}}:\right) .
$$

In principle, the gauge covariant equations of motion can be derived by requiring the BRST invariance of $W$ order by order, which in practice is quite complicated. We will therefore use a slightly different method to extract higher-order contributions of the gauge fields to the equations of motion, following closely [14, 22]. 
We start with introducing an Einbein field $e(s)$ with $d \tau=d s e(s)$ in the measure of the boundary action. This field transforms under Weyl symmetry as $\delta_{W} e=\delta \omega e$. Moreover, the one-form field also transforms nontrivially under the Weyl symmetry, with $\delta_{W} \lambda=-\delta \omega \lambda$. Then, the scalar-coupled Wilson line can be written as

$$
W=\operatorname{tr} \mathcal{P} \exp \left\{\int_{\partial \Sigma} d s e\left[N \lambda+\frac{i}{e}\left(A_{0} \partial_{s} X^{0}+A_{A^{\prime}} \partial_{s} X^{A^{\prime}}\right)\right]\right\},
$$

which is Weyl invariant classically. However, the renormalization of a classically Weyl invariant operator may lead to a Weyl anomaly. To see this, we define a renormalized operator $[\mathcal{O}(\tau)]_{r}$ for a given boundary operator $\mathcal{O}(\tau)$,

$$
[\mathcal{O}]_{r}=\exp \left[\alpha^{\prime} \int d s_{1} d s_{2} \ln d\left(s_{1}, s_{2}\right) \widehat{P}\left(s_{1}, s_{2}\right)\right] \mathcal{O},
$$

where

$$
\widehat{P}\left(s_{1}, s_{2}\right)=\frac{\delta}{\delta X^{A^{\prime}}\left(s_{1}\right)} \frac{\delta}{\delta X^{A^{\prime}\left(s_{2}\right)}}-2 i e^{-1} \partial_{s_{1}} \frac{\delta}{\delta \lambda\left(s_{1}\right)} \frac{\delta}{\delta X^{0}\left(s_{2}\right)} .
$$

Here, $d\left(s_{1}, s_{2}\right)=\int_{s_{1}}^{s_{2}} d \tau$ is the distance between $\tau\left(s_{1}\right)$ and $\tau\left(s_{2}\right)$ on $\partial \Sigma$, which transforms nontrivially under the Weyl symmetry, satisfying the following coincidence limits [22]:

$$
\left.\delta_{W} \ln d\left(s, s^{\prime}\right)\right|_{s^{\prime}=s}=\delta \omega(s),\left.\quad \delta_{W} \partial_{s} \ln d\left(s, s^{\prime}\right)\right|_{s^{\prime}=s}=\frac{1}{2} \partial_{\tau} \delta \omega(s), \quad \ldots
$$

At the lowest order of the field strength and $N$, we find

$$
\delta_{W}[W]_{r}=\alpha^{\prime} \int_{\partial \Sigma} d s e\left[\left(\lambda \delta_{W} N+i e^{-1}\left(\delta_{W} A_{0} \partial_{s} X^{0}+\delta_{W} A_{A^{\prime}} \partial_{s} X^{A^{\prime}}\right)+\cdots\right) W\right]_{r},
$$

where

$$
\begin{aligned}
\delta_{W} N & =\delta \omega D_{A^{\prime}} D^{A^{\prime}} N, \\
\delta_{W} A_{0} & =\delta \omega\left(D_{0}^{2} N-D^{A^{\prime}} E_{A^{\prime}}\right), \\
\delta_{W} A_{A^{\prime}} & =\delta \omega\left(D_{0} D_{A^{\prime}} N+D^{B^{\prime}} F_{B^{\prime} A^{\prime}}\right) .
\end{aligned}
$$

Setting $\delta_{W}[W]_{r}$ to zero at the lowest order in the field strength gives rise to the equations of motion ${ }^{12}$ of a $\mathrm{U}(n)$ Yang-Mills theory with nonrelativistic symmetry

$$
\begin{aligned}
D_{A^{\prime}} D^{A^{\prime}} N & =0, \\
D_{0}^{2} N-D^{A^{\prime}} E_{A^{\prime}} & =0, \\
D_{0} D_{A^{\prime}} N+D^{B^{\prime}} F_{B^{\prime} A^{\prime}} & =0 .
\end{aligned}
$$

These equations of motion can be derived from the following nonrelativistic Yang-Mills action:

$$
S_{\mathrm{YM}}=\frac{1}{g_{\mathrm{YM}}^{2}} \int d X^{0} d X^{A^{\prime}} \operatorname{tr}\left(\frac{1}{2} D_{0} N D_{0} N-E_{A^{\prime}} D_{A^{\prime}} N-\frac{1}{4} F_{A^{\prime} B^{\prime}} F_{A^{\prime} B^{\prime}}\right) .
$$

This nonlinear, nonabelian gauge theory has Bargmann symmetry. The corresponding equations of motion, but not the action, are invariant under the dilatation transformation $(2.18 \mathrm{e})$.

\footnotetext{
${ }^{12}$ See [23] for other versions of Yang-Mills theories with distinct nonrelativistic symmetry.
} 


\subsection{Winding open string backgrounds}

We now consider turning on open string vertex operators with nonzero winding number. This introduces novel and interesting elements in the discussion, like an emergent coordinate conjugate to the winding number, that leads to a mild nonlocality in the spacetime equations of motion, which now describe propagating degrees of freedom.

We first analyze vertex operators with a fixed winding number $w$. Focusing on the holomorphic part, the open string vertex operator (2.42) is

$$
V_{L}^{w}(z)=:\left[\frac{1}{2} N^{w} \lambda(z)-A_{0}^{w} \partial_{z} X_{L}^{0}(z)-A_{A^{\prime}}^{w} \partial_{z} X_{L}^{A^{\prime}}(z)\right] e^{i q \int^{z} d z^{\prime} \lambda\left(z^{\prime}\right)}:,
$$

where $q$ encodes the winding number $w$,

$$
q=\frac{w R}{2 \alpha^{\prime}}
$$

with $R$ the radius of the compactified $X^{1}$-direction. The new worldsheet couplings $N^{w}$, $A_{0}^{w}$, and $A_{A^{\prime}}^{w}$ are related to $N, A_{0}$, and $A_{A^{\prime}}$ by a "Fourier transform"

$$
\begin{aligned}
N\left(X^{0}, X^{A^{\prime}}, \lambda\right) & =\sum_{w} N^{w}\left(X^{0}, X^{A^{\prime}}\right) e^{i q \int^{z} d z^{\prime} \lambda\left(z^{\prime}\right)}, \\
A_{0}\left(X^{0}, X^{A^{\prime}}, \lambda\right) & =\sum_{w} A_{0}^{w}\left(X^{0}, X^{A^{\prime}}\right) e^{i q \int^{z} d z^{\prime} \lambda\left(z^{\prime}\right)}, \\
A_{A^{\prime}}\left(X^{0}, X^{A^{\prime}}, \lambda\right) & =\sum_{w} A_{A^{\prime}}^{w}\left(X^{0}, X^{A^{\prime}}\right) e^{i q \int^{z} d z^{\prime} \lambda\left(z^{\prime}\right)} .
\end{aligned}
$$

The integrated vertex operator $\mathcal{V}_{L}^{w}=-2 \int_{\partial \Sigma} d z V_{L}^{w}(z)$ is invariant under the $\mathrm{U}(1)$ gauge transformations

$$
\delta_{\epsilon} N^{w}=-2 i q \epsilon, \quad \delta_{\epsilon} A_{0}^{w}=\partial_{0} \epsilon, \quad \delta_{\epsilon} A_{A^{\prime}}^{w}=\partial_{A^{\prime}} \epsilon .
$$

We note that winding number endows $N^{w}$ with a nontrivial U(1) transformation. This fact has a simple interpretation if we introduce a novel spacetime coordinate conjugate to winding (see (2.75)). This suggests that the dynamics of wound open strings is more naturally formulated in an extended spacetime, as we shall now see.

The nonrelativistic spacetime transformations also act on $N^{w}, A_{0}^{w}$, and $A_{A^{\prime}}^{w}$ differently from the way they act on $N, A_{0}$, and $A_{A^{\prime}}$ in (2.30). For example, the boost transformation parametrized by $\Lambda^{A^{\prime}}$ in (2.30) is now modified to be

$$
\begin{aligned}
\delta_{G} N^{w} & =2 i q \Lambda_{A^{\prime}} X^{A^{\prime}} N-\Lambda^{A^{\prime}} X^{0} \partial_{A^{\prime}} N, \\
\delta_{G} A_{0}^{w} & =2 i q \Lambda_{A^{\prime}} X^{A^{\prime}} A_{0}-\Lambda^{A^{\prime}} X^{0} \partial_{A^{\prime}} A_{0}-\Lambda^{A^{\prime}} A_{A^{\prime}}, \\
\delta_{G} A_{A^{\prime}}^{w} & =2 i q \Lambda_{B^{\prime}} X^{B^{\prime}} A_{A^{\prime}}-\Lambda^{B^{\prime}} X^{0} \partial_{B^{\prime}} A_{A^{\prime}}-\Lambda_{A^{\prime}} N .
\end{aligned}
$$

The BRST transformation of $V_{L}^{w}(z)$ is given by

$$
\left[Q, V_{L}^{w}(z)\right]=: \partial_{z} W_{L}^{w}(z):-\frac{\alpha^{\prime}}{4}: \partial_{z} c(z) \mathcal{E}^{w}(z) e^{i q \int^{z} d z^{\prime} \lambda\left(z^{\prime}\right)}:
$$


with

$$
\begin{aligned}
W_{L}^{w}(z)= & c(z) V_{L}^{w}(z)+\frac{\alpha^{\prime}}{4} \partial_{z} c\left(\partial^{A^{\prime}} A_{A^{\prime}}^{w}-\partial_{0} N^{w}+2 i q A_{0}^{w}\right) e^{i q \int^{z} d z^{\prime} \lambda\left(z^{\prime}\right)} \\
\mathcal{E}^{w}(z)= & \frac{1}{2}\left(\partial^{A^{\prime}} \partial_{A^{\prime}} N^{w}+2 i q \partial_{0} N^{w}+2 i q \partial^{A^{\prime}} A_{A^{\prime}}^{w}-4 q^{2} A_{0}^{w}\right) \lambda \\
& -\left(\partial_{0}^{2} N^{w}-\partial^{A^{\prime}} E_{A^{\prime}}^{w}+2 i q \partial_{0} A_{0}^{w}\right) \partial X^{0} \\
& -\left(\partial_{0} \partial_{A^{\prime}} N^{w}+\partial^{B^{\prime}} F_{B^{\prime} A^{\prime}}^{w}+2 i q \partial_{0} A_{A^{\prime}}^{w}+2 i q E_{A^{\prime}}^{w}\right) \partial X^{A^{\prime}}
\end{aligned}
$$

where we have defined wound electric and magnetic fields

$$
E_{A^{\prime}}^{w}=\partial_{0} A_{A^{\prime}}^{w}-\partial_{A^{\prime}} A_{0}^{w}, \quad F_{A^{\prime} B^{\prime}}^{w}=\partial_{A^{\prime}} A_{B^{\prime}}^{w}-\partial_{B^{\prime}} A_{A^{\prime}}^{w} .
$$

It is useful to consider the vertex operator obtained by summing over all windings,

$$
\mathcal{V}_{L}=\sum_{w} \mathcal{V}_{L}^{w}
$$

Demanding that $\mathcal{V}_{L}=\sum_{w} \mathcal{V}_{L}^{w}$ is BRST invariant sets $\sum_{w} \mathcal{E}^{w}=0$, with $\mathcal{E}^{w}$ given in (2.71). It is enlightening to define an auxiliary coordinate,

$$
X^{r}(z) \equiv \frac{1}{2} \int^{z} d z^{\prime} \lambda\left(z^{\prime}\right)
$$

and introduce the "spacetime" coordinates $X^{\mathcal{A}}=\left(X^{r}, X^{0}, X^{A^{\prime}}\right)$. This is reminiscent of a spacetime doubling as in double field theory [24]. Using (2.71), we find that $\sum_{w} \mathcal{E}^{w}=0$ gives rise to a system of equations of motions that admits the following simple representation:

$$
G^{\mathcal{B C}} \partial_{\mathcal{B}} F_{\mathcal{C A}}=0
$$

where $F_{\mathcal{A B}}=\partial_{\mathcal{A}} A_{\mathcal{B}}-\partial_{\mathcal{B}} A_{\mathcal{A}}$, with $A_{\mathcal{A}}=\left(-N, A_{0}, A_{A^{\prime}}\right)$, and

$$
G^{\mathcal{A B}}=\left(\begin{array}{ccc}
0 & 1 & 0 \\
1 & 0 & 0 \\
0 & 0 & \delta^{A^{\prime} B^{\prime}}
\end{array}\right) .
$$

Here, $N, A_{0}$ and $A_{A^{\prime}}$ are functions of the extended spacetime coordinates $X^{\mathcal{A}}$.

The equations of motion for the open string fields with winding (2.76) can be derived by varying the following action:

$$
S=-\frac{1}{4 g^{2}} \int d X^{r} d X^{0} d X^{A^{\prime}} F_{\mathcal{A B}} G^{\mathcal{A C}} G^{\mathcal{B D}} F_{\mathcal{C D}}
$$

We note that we have been able to write down an action by introducing an auxiliary coordinate $X^{r}$, conjugate to winding number. The explicit dependence on the extended direction $X^{r}$ rather than only the local coordinates $X^{0}$ and $X^{A^{\prime}}$ signatures nonlocality. This nonlocal feature is expected as the winding sector is inherently nonlocal. This nonlocality is, however, of a rather tame form, as we are able to write down explicitly the spacetime action (2.78) in terms of the auxiliary coordinate $X^{r}$. 
In contrast to Galilean Electrodynamics, which is non-dynamical, and which describes the sector with vanishing winding, the gauge theory in (2.78) has $d-2$ propagating degrees of freedom with a nonrelativistic dispersion relation,

$$
p_{0}=\alpha^{\prime} \frac{p_{A^{\prime}} p_{A^{\prime}}}{2 w R} .
$$

This beautifully realizes the nonrelativistic open string dispersion relation in (2.13) with the open string excitation number $N_{\text {open }}$ set to zero.

The compact form of the equations of motion in (2.76) finds a simple interpretation if one performs a T-duality transformation along the longitudinal spatial $X^{1}$-direction, which is compactified on a circle of radius $R$. After performing this longitudinal spatial T-duality transformation in nonrelativistic open string theory, we find relativistic open string theory on a spacetime-filling brane background, with the following duality dictionary [25]: the $X^{1}$-direction is dual to a lightlike direction $\widetilde{X}^{1}=X^{r}$ on the brane, the Goldstone boson $N$ and the gauge component $A_{0}$ are dual to the lightlike components of the relativistic $\mathrm{U}(1)$ gauge field, and the winding number $w$ is dual to the discrete momentum along the lightlike circle.

This concludes our analysis of nonrelativistic open string theory in flat spacetime.

\section{Curved backgrounds}

In this section, we consider the nonrelativistic string nonlinear sigma model in an arbitrary string Newton-Cartan geometry, $B$-field, U(1) gauge field, and dilaton background in presence of a $\mathrm{D}(d-2)$-brane. Our goal is to compute the beta-functions for the open string couplings, which define the spacetime equations of motion of nonrelativistic open string theory. We focus our discussion to the zero winding sector. We start with a brief review of the nonrelativistic closed string nonlinear sigma model, and then move on to the construction of the Dirichlet nonlinear sigma model that couples nonrelativistic open strings to a closed string background geometry.

\subsection{Closed strings on a string Newton-Cartan background}

The appropriate closed string background geometry for nonrelativistic string theory is string Newton-Cartan geometry, which is a non-Lorentzian and non-Riemannian geometry. We give a short review of the definition of string Newton-Cartan geometry, following closely [12].

Let $\mathcal{T}_{p}$ be the tangent space attached to a point $p$ in the spacetime $\mathcal{M}$. We decompose $\mathcal{T}_{p}$ into a longitudinal sector with an index $A=0,1$ and a transverse sector with an index $A^{\prime}=2, \cdots, d-1$. We introduce a longitudinal Vielbein field $\tau_{\mu}{ }^{A}$ and a transverse Vielbein field $E_{\mu}{ }^{A^{\prime}}$. The invertibility conditions are

$$
\begin{array}{rlrl}
\tau^{\mu}{ }_{A} \tau_{\mu}{ }^{B} & =\delta_{A}^{B}, & \tau_{\mu}{ }^{A} \tau_{A}^{\nu}+E_{\mu}{ }^{A^{\prime}} E^{\nu}{ }_{A^{\prime}} & =\delta_{\mu}^{\nu}, \\
E^{\mu}{ }_{A^{\prime}} E_{\mu}{ }^{B^{\prime}} & =\delta_{A^{\prime}}^{B^{\prime}}, & \tau^{\mu}{ }_{A} E_{\mu}{ }^{A^{\prime}}=E^{\mu}{ }_{A^{\prime}} \tau_{\mu}{ }^{A}=0 .
\end{array}
$$


In addition, there is an additional gauge field $m_{\mu}{ }^{A}$, associated with a noncentral extension in the string Newton-Cartan algebra.

The string Newton-Cartan geometry realizes the string Newton-Cartan algebra [12] as a gauge symmetry acting on the target space that we will define momentarily. This string Newton-Cartan algebra is a finite subalgebra of the extended Galilean symmetry algebra discussed in section 2.2 for the flat spacetime free action in (2.1) in the absence of a boundary. Turning on an arbitrary closed string background breaks the infinitedimensional extended Galilean symmetry algebra to the finite-dimensional string NewtonCartan symmetry algebra, ${ }^{13}$ whose generators consist of a longitudinal translation $H_{A}$, a transverse translation $P_{A^{\prime}}$, a longitudinal Lorentz rotation $M$, a string Galilean boost $G_{A A^{\prime}}$, a transverse rotation $J_{A^{\prime} B^{\prime}}$, and noncentral extensions $Z_{A}$ and $Z_{A B}$, with $\eta^{A B} Z_{A B}=0$ and $\eta^{A B}$ the Minkowski metric. The string Newton-Cartan gauge fields transform under the string Newton-Cartan symmetry as [12, 26]

$$
\begin{aligned}
\delta \tau_{\mu}{ }^{A} & =\Lambda \epsilon^{A}{ }_{B} \tau_{\mu}{ }^{B}, \quad \delta E_{\mu}{ }^{A^{\prime}}=-\Lambda_{A}{ }^{A^{\prime}} \tau_{\mu}{ }^{A}+\Lambda^{A^{\prime}{ }_{B^{\prime}} E_{\mu}{ }^{B^{\prime}},} \\
\delta m_{\mu}{ }^{A} & =D_{\mu} \sigma^{A}+\Lambda \epsilon_{B}^{A} m_{\mu}{ }^{B}+\Lambda^{A A^{\prime}} E_{\mu}{ }^{A^{\prime}}-\tau_{\mu}{ }^{B} \sigma^{A}{ }_{B},
\end{aligned}
$$

where $\sigma^{A B}$ is traceless with $\sigma^{A B} \eta_{A B}=0$ and $D_{\mu} \sigma^{A}=\partial_{\mu} \sigma^{A}-\epsilon_{B}^{A} \sigma^{B} \Omega_{\mu}$ with $\Omega_{\mu}$ the spin connection associated with the longitudinal Lorentz rotation. The Levi-Civita symbol $\epsilon_{A B}$ is defined by $\epsilon_{01}=-\epsilon_{10}=1$, and the $A$ index can be raised by the Minkowskian metric $\eta^{A B}$. Here, the Lie group parameter $\Lambda$ is associated with $M, \Lambda^{A A^{\prime}}$ is associated with $G_{A A^{\prime}}, \Lambda^{A^{\prime} B^{\prime}}$ is associated with $J_{A^{\prime} B^{\prime}}, \sigma^{A}$ is associated with $Z_{A}$, and $\sigma^{A B}$ is associated with $Z_{A B}$. Here, we omitted the symmetry transformations under translations and, instead, require that all gauge fields transform as covariant vectors under diffeomorphisms. These generators satisfy the string Newton-Cartan algebra with the following Lie brackets:

$$
\begin{array}{rlrl}
{\left[H_{A}, G_{B A^{\prime}}\right]} & =\eta_{A B} P_{A^{\prime}}, & {\left[H_{A}, Z\right]} & =\epsilon_{A}^{B} Z_{B}, \\
{\left[G_{A A^{\prime}}, P_{B^{\prime}}\right]} & =\delta_{A^{\prime} B^{\prime}} Z_{A}, & {\left[H_{A}, Z_{B C}\right]} & =2 \eta_{A C} Z_{B}-\eta_{B C} Z_{A}, \\
{\left[G_{A A^{\prime}}, G_{B B^{\prime}}\right]} & =\delta_{A^{\prime} B^{\prime}} Z_{[A B]} .
\end{array}
$$

Here, we omitted the Lie brackets that involve the longitudinal and transverse rotational generators $M_{A B}=M \epsilon_{A B}$ and $J_{A^{\prime} B^{\prime}}$, which act on the $A$ and $A^{\prime}$ indices in a standard way, for example, as in (2.21).

Next, we consider the nonlinear sigma model in a string Newton-Cartan background. For now, we set the worldsheet to be flat and thus omit the dilaton field. We will return to the dilaton contribution at the end in section 3.5. The nonlinear sigma model of nonrelativistic closed string theory in an arbitrary string Newton-Cartan background with a Kalb-Ramond field $B_{\mu \nu}$ is

$$
\begin{aligned}
S_{\text {closed }} & =\frac{1}{4 \pi \alpha^{\prime}} \int d^{2} \sigma\left(\partial_{\alpha} X^{\mu} \partial^{\alpha} X^{\nu} H_{\mu \nu}+\lambda \bar{\partial} X^{\mu} \tau_{\mu}+\bar{\lambda} \partial X^{\mu} \bar{\tau}_{\mu}-i \epsilon^{\alpha \beta} \partial_{\alpha} X^{\mu} \partial_{\beta} X^{\nu} B_{\mu \nu}\right), \\
H_{\mu \nu} & =E_{\mu}{ }^{A^{\prime}} E_{\nu}{ }^{A^{\prime}}+\left(\tau_{\mu}{ }^{A} m_{\nu}{ }^{B}+\tau_{\nu}{ }^{A} m_{\mu}{ }^{B}\right) \eta_{A B}
\end{aligned}
$$

\footnotetext{
${ }^{13}$ The string Newton-Cartan algebra can be further extended by including a longitudinal dilatation generator, similarly as in (2.23) for the Bargmann algebra. See [12] for discussion on this dilatational generator.
} 
where $\partial=\partial_{\sigma}-i \partial_{\tau}$ and $\bar{\partial}=\partial_{\sigma}+i \partial_{\tau}$ and the Levi-Civita symbol $\epsilon^{\alpha \beta}$ is defined by $\epsilon^{\tau \sigma}=-\epsilon^{\sigma \tau}=1$. The $B$-field transforms under an $\mathrm{U}(1)$ gauge symmetry

$$
\delta_{\epsilon} B_{\mu \nu}=\partial_{\mu} \epsilon_{\nu}-\partial_{\nu} \epsilon_{\mu} .
$$

The two-tensor $H_{\mu \nu}$ is invariant under the string Galilean boost but not the $Z_{A}$ extension. For $S_{\text {closed }}$ to be invariant under the $Z_{A}$ symmetry that acts on $m_{\mu}{ }^{A}$ as in (3.2), it is required that a hypersurface orthogonality condition is satisfied,

$$
D_{[\mu} \tau_{\nu]}^{A}=0 .
$$

With the condition (3.6) taken into account, the action (3.4) is invariant under the gauge transformations (3.2) and (3.5). The $Z_{A}$ gauge symmetry we consider here prohibits the $\lambda \bar{\lambda}$ operator from being generated at the quantum level in the string action (3.4). Turning on such a $\lambda \bar{\lambda}$ term would drive the nonlinear sigma model towards the one that describes relativistic string theory. See $[14,27]$ for details. ${ }^{14}$

Sometimes it is useful to rewrite the string action using the field redefinitions [7]

$$
\lambda^{\prime}=C^{-1}\left(\lambda-\partial X^{\mu} \bar{C}_{\mu}\right), \quad \bar{\lambda}^{\prime}=\bar{C}^{-1}\left(\bar{\lambda}-\bar{\partial} X^{\mu} C_{\mu}\right),
$$

and

$$
\begin{aligned}
\tau_{\mu}^{\prime}=C \tau_{\mu}, & H_{\mu \nu}^{\prime}=H_{\mu \nu}-\left(C_{\mu}{ }^{A} \tau_{\nu}{ }^{A}+C_{\nu}{ }^{A} \tau_{\mu}{ }^{B}\right) \eta_{A B}, \\
\bar{\tau}_{\mu}=\bar{C} \bar{\tau}_{\mu}, & B_{\mu \nu}^{\prime}=B_{\mu \nu}+\left(C_{\mu}{ }^{A} \tau_{\nu}{ }^{B}-C_{\nu}{ }^{A} \tau_{\mu}{ }^{B}\right) \epsilon_{A B} .
\end{aligned}
$$

This can be thought of as a Stueckelberg symmetry in (3.4). Here, $C_{\mu}=C_{\mu}{ }^{0}+C_{\mu}{ }^{1}$ and $\bar{C}_{\mu}=C_{\mu}{ }^{0}-C_{\nu}{ }^{1}$. The parameters $C, \bar{C}$ and $C_{\mu}{ }^{A}$ are arbitrary functions of $X^{\mu}$ satisfying the condition $E_{A^{\prime}}^{\mu} \partial_{\mu}(C \bar{C})=0$. The dilaton field $\Phi$, when included, will also receive a redefinition,

$$
\Phi^{\prime}=\Phi+\frac{1}{2} \ln (\bar{C} C)
$$

\subsection{Dirichlet sigma model for nonrelativistic open strings}

We now consider nonrelativistic string theory defined by the worldsheet action (3.4) with a boundary $\partial \Sigma$ at $\sigma=0$ on the worldsheet $\Sigma$ and the boundary conditions corresponding to a $\mathrm{D}(d-2)$-brane, described by a codimension-one submanifold $\mathcal{N}$ embedded in the target space $\mathcal{M}$. In a curved spacetime, we take

$$
\left.X^{\mu}\right|_{\sigma=0}=f^{\mu}\left(Y^{i}\right)
$$

\footnotetext{
${ }^{14}$ Note that $B_{\mu \nu}$ is taken to be invariant under the $Z_{A}$ gauge transformation here. In [13], a different spacetime gauge symmetry group is proposed, in which there is a different $Z_{A}$ symmetry transformation that acts nontrivially on both $m_{\mu}{ }^{A}$ and $B_{\mu \nu}$, and the condition (3.6) is not imposed classically. In this latter case, a $\lambda \bar{\lambda}$ term in the sigma model will in general be generated by quantum corrections [14]. However, it is possible to relax the condition (3.6) to be $E^{\mu}{ }_{A^{\prime}} E^{\nu}{ }_{B^{\prime}} D_{[\mu} \tau_{\nu]}{ }^{A}=0$ without generating a $\lambda \bar{\lambda}$ operator at all loops in the two-dimensional worldsheet sigma model (which can be shown, for example, by using the method in [27]). The condition $E^{\mu}{ }_{A^{\prime}} E^{\nu}{ }_{B^{\prime}} D_{[\mu} \tau_{\nu]}{ }^{A}=0$ is related to the twistless torsion conditions explored in [28]. There is no known symmetry reasoning for this type of twistless torsion conditions to hold from the worldsheet perspective.
} 
where $Y^{i}, i=0,1, \cdots, d-2$ are coordinates in the submanifold $\mathcal{N}$. The function $f^{\mu}$ in (3.9) describes how the $\mathrm{D}(d-2)$-brane is embedded in the $d$-dimensional spacetime. The $d$ conditions in (3.9) imply that

$$
\left.\delta X^{\mu}\right|_{\sigma=0}=\delta Y^{i} \partial_{i} f^{\mu}(Y)
$$

i.e., $\left.\delta X^{\mu}\right|_{\partial \Sigma}$ is tangent to $\mathcal{N}$. The boundary condition (3.9) generalizes the Dirichlet boundary condition (2.8) in flat spacetime.

Unbroken phase. The D-brane submanifold $\mathcal{N}$ spontaneously breaks the string NewtonCartan symmetry group generated by (3.3) to the Bargmann symmetry group generated by (2.21). This theory can be studied in the unbroken and broken phase. The NambuGoldstone boson associated with this spontaneous symmetry breaking is the massless mode associated with perturbing the shape of the brane, which can be thought of as part of $f^{\mu}$ by allowing the brane to fluctuate. This defines the unbroken phase of the theory.

In the unbroken phase, we introduce the background fields for open strings on the D-brane by exponentiating the vertex operator in (2.28), which modifies the closed string action (3.4) to be

$$
\begin{aligned}
S= & \frac{1}{4 \pi \alpha^{\prime}} \int_{\Sigma} d^{2} \sigma\left(\partial_{\alpha} X^{\mu} \partial^{\alpha} X^{\nu} H_{\mu \nu}+\lambda \bar{\partial} X^{\mu} \tau_{\mu}+\bar{\lambda} \partial X^{\mu} \bar{\tau}_{\mu}-i \epsilon^{\alpha \beta} \partial_{\alpha} X^{\mu} \partial_{\beta} X^{\nu} B_{\mu \nu}\right) \\
& +\frac{1}{2 \pi \alpha^{\prime}} \int_{\partial \Sigma} d \tau\left[\frac{1}{2} N(Y)(\lambda-\bar{\lambda})+i A_{i}(Y) \partial_{\tau} Y^{i}\right] .
\end{aligned}
$$

The definitions of the boundary background fields $N$ and $A_{i}$ here are the same as in flat spacetime up to a rescaling factor $2 \pi \alpha^{\prime}$. Varying $\lambda$ and $\bar{\lambda}$ on the boundary sets

$$
N(Y)=0 \text {. }
$$

Furthermore, using (3.9) and varying the total action with respect to the coordinates $Y^{i}$ on the brane, we find $d-1$ boundary conditions that generalize the Neumann boundary conditions (2.10) and (2.12) in flat spacetime,

$$
\partial_{i} f^{\mu} H_{\mu \nu} \partial_{\sigma} X^{\nu}+i \mathcal{F}_{i j} \partial_{\tau} X^{j}+\frac{1}{2} \partial_{i} f^{\mu}\left(\lambda \tau_{\mu}+\bar{\lambda} \bar{\tau}_{\mu}\right)=0,
$$

where $\mathcal{F}_{i j} \equiv b_{i j}+F_{i j}$, with $b_{i j} \equiv \partial_{i} f^{\mu} B_{\mu \nu} \partial_{j} f^{\nu}$ and $F_{i j} \equiv \partial_{i} A_{j}-\partial_{j} A_{i}$. Finally, the Lagrange multipliers $\lambda$ and $\bar{\lambda}$ impose the bulk equations of motion

$$
\bar{\partial} X^{\mu} \tau_{\mu}=0, \quad \partial X^{\mu} \bar{\tau}_{\mu}=0,
$$

which upon restricting to the boundary give the boundary conditions

$$
\partial_{\sigma} X^{\mu} \tau_{\mu}{ }^{A}=i \epsilon_{B}^{A} \partial_{\tau} Y^{i} \partial_{i} f^{\mu} \tau_{\mu}{ }^{B} .
$$

In the unbroken phase, we choose to expand around the reference configuration defined by the boundary conditions (3.12)-(3.15) with $N=0$, and $N$ decouples from any further calculation on the beta-functions. However, there will still be counterterms generated for 
$N$, which will give rise to a nontrivial beta function $\beta(N)$. This is similar to the situation in relativistic open string theory [2].

The full string Newton-Cartan symmetry in (3.2) is preserved by the boundary conditions (3.12)-(3.15) in this unbroken phase, provided that $\lambda, \bar{\lambda}$ transform on the brane as $[12]$

$$
\begin{aligned}
& \left.\delta \lambda\right|_{\sigma=0}=\Lambda \lambda+\partial X^{\mu}\left[D_{\mu}\left(\sigma^{0}-\sigma^{1}\right)+\tau_{\mu}\left(\sigma^{00}-\sigma^{(01)}\right)\right] \\
& \left.\delta \bar{\lambda}\right|_{\sigma=0}=-\Lambda \bar{\lambda}+\bar{\partial} X^{\mu}\left[D_{\mu}\left(\sigma^{0}+\sigma^{1}\right)+\bar{\tau}_{\mu}\left(\sigma^{00}+\sigma^{(01)}\right)\right]
\end{aligned}
$$

and that $A_{i}$ transforms as

$$
\delta A_{i}=-\epsilon_{A B} \sigma^{A} \partial_{i} f^{\mu} \tau_{\mu}{ }^{B}
$$

The Stueckelberg symmetry in (3.7), with a trivial action on $A_{i}$, is also preserved by the boundary conditions (3.12)-(3.15).

Broken phase. Next, we consider the uniformly broken phase where the vacuum expectation value for $f^{\mu}$ is

$$
\left\langle f^{\mu}\left(Y^{i}\right)\right\rangle=f_{0}^{\mu}\left(Y^{i}\right)
$$

with $f_{0}^{\mu}$ a fixed embedding function that contains no massless excitations. Define a coordinate system $X^{\mu}=\left(y, Y^{i}\right)$ adapted to the submanifold specified by the embedding function $f_{0}^{\mu}\left(Y^{i}\right)$, with

$$
f_{0}^{y}=y_{0}, \quad f_{0}^{i}=Y^{i},
$$

where $y_{0}$ is a fixed location in the $y$ direction that determines where the brane is. We only need this set of adapted coordinates to be defined in a neighborhood of the submanifold. In nonrelativistic open string theory, we need to take the boundary values

$$
\tau_{y}^{0}=E_{y}^{A^{\prime}}=0, \quad \tau_{y}{ }^{1} \neq 0,
$$

which naturally generalize the requirement that the submanifold is transverse to the $X^{1}$ direction in flat spacetime. It is convenient to rescale $\lambda$ and $\bar{\lambda}$ in (3.11) to normalize $\tau_{y}{ }^{1}=1$ on the boundary. Applying these prescriptions to the invertibility conditions in (3.1), we find on the boundary that

$$
\tau^{y}{ }_{1}=1, \quad \tau^{i}{ }_{1}=0, \quad \tau^{y}{ }_{0}=-\tau_{0}^{i} \tau_{i}{ }^{1}, \quad E^{y}{ }_{A^{\prime}}=-E^{i}{ }_{A^{\prime}} \tau_{i}{ }^{1},
$$

and

$$
\begin{aligned}
& \tau_{0}^{i} \tau_{i}^{0}=1, \\
& \tau_{i}{ }^{0} \tau^{j}{ }_{0}+E_{i}{ }^{A^{\prime}} E^{j}{ }_{A^{\prime}}=\delta_{i}^{j}, \\
& E^{i}{ }_{A^{\prime}} E_{i}^{B^{\prime}}=\delta_{A^{\prime}}^{B^{\prime}}, \\
& \tau_{0}^{i} E_{i}{ }^{A^{\prime}}=E^{i}{ }_{A^{\prime}} \tau_{i}{ }^{0}=0 \text {. }
\end{aligned}
$$

Now, we consider the massless excitation that perturbs around the vacuum expectation value $f_{0}^{\mu}\left(Y^{i}\right)$. We parametrize $f^{\mu}\left(Y^{i}\right)$ as

$$
f^{y}\left(Y^{i}\right)=y_{0}+\pi\left(Y^{i}\right), \quad f^{i}\left(Y^{i}\right)=Y^{i}
$$


where $\pi\left(Y^{i}\right)$ is the Nambu-Goldstone boson that perturbs around the brane. In nonrelativistic open string theory, it is possible to take a change of variables that is discontinuous on the boundary, under which $\pi\left(Y^{i}\right)$ contributes a $(\lambda-\bar{\lambda})$-term to the boundary action in (3.11) and thus gives rise to a nonzero $N=\pi .^{15}$

The chosen vacuum expectation value of $f^{\mu}$ preserves the time translation generated by $H_{0}$, the transverse spatial translation generated by $P_{A^{\prime}}$, the Galilean boost generated by $G_{0 A^{\prime}}$, and the transverse rotation generated by $J_{A^{\prime} B^{\prime}}$. These generators form the Bargmann algebra in (2.21). ${ }^{16}$ In contrast, the translational symmetry generated by $H_{1}$, the longitudinal Lorentz rotation generated by $M$, and the part of the string Galilean boost symmetry generated by $G_{1 A^{\prime}}$ are broken on the boundary. This can be seen by requiring the transformations in (3.2) (with translations included) to preserve the boundary values in (3.20).

In the following, we will proceed with the calculation of the beta-functions for the Dirichlet nonlinear sigma model (3.11). We will mostly focus on the unbroken phase formalism, where the calculation is simpler. The broken phase formalism will be useful later when we vary the worldvolume DBI-like action to derive the equations of motion on the brane.

\subsection{Covariant background field method}

In the rest of the section, we compute the beta-functions of the coupling $A_{i}$ and $N$ in the 2d QFT (3.11) around the classical configuration $N(Y)=0$. We start with rewriting (3.11) by using the field redefinition (3.7) with $A_{i}^{\prime}=A_{i}, C=\bar{C}=1$ and $C_{\mu}{ }^{A}=m_{\mu}{ }^{A}$, which gives rise to the following equivalent action:

$$
\begin{aligned}
S= & \frac{1}{4 \pi \alpha^{\prime}} \int_{\Sigma} d^{2} \sigma\left(\partial_{\alpha} X^{\mu} \partial^{\alpha} X^{\nu} E_{\mu \nu}+\lambda \bar{\partial} X^{\mu} \tau_{\mu}+\bar{\lambda} \partial X^{\mu} \bar{\tau}_{\mu}-i \epsilon^{\alpha \beta} \partial_{\alpha} X^{\mu} \partial_{\beta} X^{\nu} \mathscr{B}_{\mu \nu}\right) \\
& +\frac{1}{2 \pi \alpha^{\prime}} \int_{\partial \Sigma} d \tau\left[\frac{1}{2} N(Y)(\lambda-\bar{\lambda})+i A_{i}(Y) \partial_{\tau} Y^{i}\right]
\end{aligned}
$$

where

$$
E_{\mu \nu}=E_{\mu}{ }^{A^{\prime}} E_{\nu}{ }^{A^{\prime}}, \quad \mathscr{B}_{\mu \nu}=B_{\mu \nu}+\left(m_{\mu}{ }^{A} \tau_{\nu}{ }^{B}-m_{\nu}{ }^{A} \tau_{\mu}{ }^{B}\right) \epsilon_{A B}
$$

In this way of rewriting, $E_{\mu \nu}$ is invariant under the $Z_{A}$ symmetry but not invariant under the boost symmetry. This action is supplemented with the classical boundary conditions (3.12)-(3.15), which after the field redefinitions become

$$
\begin{aligned}
N=0, \quad \tau_{\mu}{ }^{A} \partial_{\sigma} X^{\mu} & =i \epsilon_{B}^{A} \partial_{\tau} Y^{i} t_{i}{ }^{B}, \\
E_{\mu}{ }^{A^{\prime}} \partial_{\sigma} X^{\mu} & =-e_{A^{\prime}}^{i}\left[i \mathscr{F}_{i j} \partial_{\tau} Y^{j}+\frac{1}{2}(\lambda-\bar{\lambda}) t_{i}{ }^{1}\right], \\
\frac{1}{2}(\lambda+\bar{\lambda}) & =-t_{0}^{i}{ }_{0}\left[i \mathscr{F}_{i j} \partial_{\tau} Y^{j}+\frac{1}{2}(\lambda-\bar{\lambda}) t_{i}{ }^{1}\right] .
\end{aligned}
$$

Here, we defined the projections

$$
t_{i}{ }^{A}=\partial_{i} f^{\mu} \tau_{\mu}{ }^{A}, \quad e_{i}^{A^{\prime}}=\partial_{i} f^{\mu} E_{\mu}{ }^{A^{\prime}}, \quad \mathscr{F}_{i j}=\partial_{i} f^{\mu} \mathscr{B}_{\mu \nu} \partial_{j} f^{\nu}+F_{i j},
$$

\footnotetext{
${ }^{15}$ See similar discussions for relativistic open string theory in [2, 29]. Also see [25] for further details.

${ }^{16}$ The central charge $Z$ will be generated by commuting $P_{A^{\prime}}$ and $G_{0 A^{\prime}}$.
} 
where $F_{i j}=\partial_{i} A_{j}-\partial_{j} A_{i}$. The inverse Vielbeine fields $e^{i} A^{\prime}$ and $t_{0}^{i}$ are defined via the invertibility conditions on the brane,

$$
\begin{array}{rlrl}
t_{0}^{i} t_{i}{ }^{0} & =1, & t_{i}{ }^{0} t^{j}{ }_{0}+e_{i}{ }^{A^{\prime}} e^{j}{ }_{A^{\prime}} & =\delta_{i}^{j}, \\
e^{i}{ }_{A^{\prime}} e_{i}{ }^{B^{\prime}} & =\delta_{A^{\prime}}^{B^{\prime}}, & t_{0}^{i} e_{i}{ }^{A^{\prime}}=e^{i}{ }_{A^{\prime}} t_{i}{ }^{0}=0 .
\end{array}
$$

Comparing with (3.1), we find the consistency conditions

$$
\tau^{\mu}{ }_{0}=\partial_{i} f^{\mu} t_{0}^{i}, \quad E^{\mu}{ }_{A^{\prime}}=\partial_{i} f^{\mu} e_{A^{\prime}}^{i} .
$$

Next, we expand the sigma model (3.24) with respect to quantum fluctuations using the background field method, around a covariant background that satisfies the classical configurations (3.12)-(3.15).

Consider a sufficiently small neighborhood $\mathcal{O}_{\mathcal{M}}$ of a point $X_{0}^{\mu}$ in the target space $\mathcal{M}$. For an arbitrary point $X^{\mu}$ in $\mathcal{O}_{\mathcal{M}}$, there exists a unique geodesic in $\mathcal{M}$ interpolating between $X_{0}^{\mu}$ and $X^{\mu}$, parametrized by $X_{u}^{\mu}$, with an affine parameter $u \in[0,1]$, such that

$$
\frac{d^{2} X_{u}^{\mu}}{d u^{2}}+\Gamma_{\rho \sigma}^{\mu} \frac{d X_{u}^{\rho}}{d u} \frac{d X_{u}^{\sigma}}{d u}=0 .
$$

We require $X_{u=0}=X_{0}^{\mu}$ and $X_{u=1}=X^{\mu}$. Here, $\Gamma_{\rho \sigma}^{\mu}$ is the Christoffel symbol in string Newton-Cartan geometry. ${ }^{17}$ Define the covariant quantum fluctuation

$$
\xi^{\mu}=\left.\frac{d X_{u}^{\mu}}{d u}\right|_{u=0}
$$

which is the tangent vector to the geodesic at $u=0$. Similarly, consider a sufficiently small neighborhood $\mathcal{O}_{\mathcal{N}}$ of a point $Y_{0}^{i}$ in the submanifold $\mathcal{N}$. For an arbitrary point $Y^{i}$ in $\mathcal{O}_{\mathcal{N}}$, there exists a unique geodesic in $\mathcal{N}$ interpolating between $Y_{0}^{i}$ and $Y^{i}$, parametrized by $Y_{v}^{i}$, with an affine parameter $v \in[0,1]$, such that

$$
\frac{d^{2} Y_{v}^{i}}{d v^{2}}+\Gamma^{i}{ }_{j k} \frac{d Y_{v}^{j}}{d v} \frac{d Y_{v}^{k}}{d v}=0
$$

We require $Y_{v=0}=Y_{0}^{i}$ and $Y_{v=1}=Y^{i}$. Here, $\Gamma^{i}{ }_{j k}$ is the Christoffel symbol in NewtonCartan geometry on the brane. Define the covariant quantum fluctuation

$$
\zeta^{i}=\left.\frac{d Y_{v}^{i}}{d v}\right|_{v=0},
$$

which is the tangent vector to the geodesic at $v=0$. The tangent vector $\zeta^{i}(Y)$ on the brane submanifold $\mathcal{N}$ is related to the tangent vector $\xi^{\mu}(X)$ in $\mathcal{M}$ as follows [2]:

$$
\left.\xi^{\mu}\right|_{\sigma=0}=\zeta^{i} \partial_{i} f^{\mu}+\frac{1}{2} \zeta^{i} \zeta^{j} K^{\mu}{ }_{i j}+\cdots,
$$

where $K^{\mu}{ }_{i j}$ is the extrinsic curvature of the brane submanifold, defined by

$$
K_{i j}^{\mu}=\nabla_{i} \nabla_{j} f^{\mu}+\Gamma_{\rho \sigma}^{\mu} \partial_{i} f^{\rho} \partial_{j} f^{\sigma} .
$$

\footnotetext{
${ }^{17}$ The explicit form of $\Gamma_{\rho \sigma}^{\mu}$ is given in [10] but with $m_{\mu}{ }^{A}$ set to zero, since the dependence on $m_{\mu}{ }^{A}$ has been relocated to be in $\mathscr{B}_{\mu \nu}$.
} 
Here, $\nabla_{i}$ is a Newton-Cartan covariant derivative in the submanifold $\mathcal{N}$ and $\Gamma^{\mu}{ }_{\rho \sigma}$ is the Christoffel symbol in string Newton-Cartan geometry, with

$$
\left.\Gamma_{\rho \sigma}^{\mu}\right|_{\sigma=0}=\tau_{A}^{\mu} \partial_{(\rho \sigma)}{ }^{A}+E^{\mu}{ }_{A^{\prime}}\left[\partial_{(\rho} E_{\sigma)}{ }^{A^{\prime}}-\Omega_{(\rho}{ }^{A^{\prime} B^{\prime}} E_{\sigma)}{ }^{B^{\prime}}-\Omega_{(\rho}{ }^{0 A^{\prime}} \tau_{\sigma)}{ }^{0}\right],
$$

where $\Omega_{\mu}{ }^{A A^{\prime}}$ is the spin connection associated with the string Galilean boost and $\Omega_{\mu}{ }^{A^{\prime} B^{\prime}}$ is the spin connection associated with the transverse rotation. We used that $\left.\Omega_{\mu}{ }^{A B}\right|_{\sigma=0}=$ $\left.\Omega_{\mu}{ }^{1 A^{\prime}}\right|_{\sigma=0}=0$ since they are associated with spontaneously broken symmetries, where $\Omega_{\mu}{ }^{A B}$ is the spin connection associated with the longitudinal Lorentz boost. In the adapted coordinates (3.23), we also have the boundary conditions $\left.\Omega_{y}{ }^{0 A^{\prime}}\right|_{\sigma=0}=\left.\Omega_{y}{ }^{A^{\prime} B^{\prime}}\right|_{\sigma=0}=0$. It then follows that, on the boundary,

$$
E_{\mu}{ }^{A^{\prime}} K^{\mu}{ }_{i j}=\tau_{\mu}{ }^{0} K^{\mu}{ }_{i j}=0, \quad \tau_{\mu}{ }^{1} K^{\mu}{ }_{i j}=\nabla_{i} t_{j}{ }^{1}=\nabla_{j} t_{i}{ }^{1} .
$$

A covariant expansion with respect to $\xi^{\mu}(X)$ for the closed string action, i.e. the bulk part in (3.24), has been put forward in [27], where $\lambda$ and $\bar{\lambda}$ are also split into a classical and quantum part as

$$
\lambda=\lambda_{0}+\rho, \quad \bar{\lambda}=\bar{\lambda}_{0}+\rho .
$$

By incorporating a covariant expansion with respect to $\zeta^{i}(Y)$ for the boundary action in (3.24), in a similar way as in [2], we find the expanded action to be

$$
S[\lambda, \bar{\lambda}, X, Y]=S^{(0)}+S^{(1)}+S^{(2)}+O(\rho, \bar{\rho}, \xi, \zeta)^{3},
$$

where $S^{(0)}=S\left[\lambda_{0}, \bar{\lambda}_{0}, X_{0}, Y_{0}\right]$ and

$$
\begin{aligned}
& S^{(1)}= \frac{1}{4 \pi \alpha^{\prime}} \int_{\Sigma} d^{2} \sigma\left\{\xi ^ { \rho } \left[\partial_{\alpha} X_{0}^{\mu} \partial^{\alpha} X_{0}^{\nu} \nabla_{\rho} E_{\mu \nu}-2 \nabla_{\alpha}\left(\partial^{\alpha} X_{0}^{\mu} E_{\rho \mu}\right)-i \epsilon^{\alpha \beta} \partial_{\alpha} X_{0}^{\mu} \partial_{\beta} X_{0}^{\nu} \mathscr{H}_{\mu \nu \rho}\right.\right. \\
&\left.\left.-\bar{\nabla}\left(\lambda_{0} \tau_{\rho}\right)-\nabla\left(\bar{\lambda}_{0} \bar{\tau}_{\rho}\right)\right]+\rho \bar{\partial} X_{0}^{\mu} \tau_{\mu}+\bar{\rho} \partial X_{0}^{\mu} \bar{\tau}_{\mu}\right\} 1 \\
&+\frac{1}{2 \pi \alpha^{\prime}} \int_{\partial \Sigma} d \tau\left\{\xi^{\mu}\left[\partial_{\sigma} X_{0}^{\nu} E_{\mu \nu}+i \partial_{\tau} Y_{0}^{i} \mathscr{B}_{\mu i}+\frac{1}{2}\left(\lambda_{0} \tau_{\mu}+\bar{\lambda}_{0} \bar{\tau}_{\mu}\right)\right]+i \zeta^{i} \partial_{\tau} Y_{0}^{j} F_{i j}\right\}, \\
& S^{(2)=} \frac{1}{4 \pi \alpha^{\prime}} \int_{\Sigma} d^{2} \sigma\left\{\nabla_{\alpha} \xi^{\mu} \nabla^{\alpha} \xi^{\nu} E_{\mu \nu}-\xi^{\mu}\left[\bar{\nabla}\left(\rho \tau_{\mu}\right)+\nabla\left(\bar{\rho} \bar{\tau}_{\mu}\right)\right]\right. \\
&+2 \xi^{\rho} \nabla_{\alpha} \xi^{\sigma}\left(\partial^{\alpha} X_{0}^{\mu} \nabla_{[\rho} E_{\sigma] \mu}-\frac{i}{2} \epsilon^{\alpha \beta} \partial_{\beta} X_{0}^{\mu} \mathscr{H}_{\mu \rho \sigma}\right) \\
&+\frac{1}{2} \xi^{\rho} \xi^{\sigma} \partial_{\alpha} X_{0}^{\mu} \partial_{\beta} X_{0}^{\nu}\left[\delta^{\alpha \beta}\left(\nabla_{\rho} \nabla_{\sigma} E_{\mu \nu}-2 \nabla_{\mu} \nabla_{\rho} E_{\sigma \nu}\right)-i \epsilon^{\alpha \beta} \nabla_{\rho} \mathscr{H}_{\sigma \mu \nu}\right] \\
&\left.+\xi^{\rho} \xi^{\sigma}\left[\partial_{\alpha} X_{0}^{\mu} \partial^{\alpha} X_{0}^{\nu} E_{\mu \lambda} R_{\rho \sigma \nu}^{\lambda}+\frac{1}{2}\left(\lambda_{0} \bar{\partial} X_{0}^{\mu} \tau_{\kappa}+\bar{\lambda}_{0} \partial X_{0}^{\mu} \bar{\tau}_{\kappa}\right) R_{\rho \sigma \mu}^{\kappa}\right]\right\} \\
&+\frac{1}{4 \pi \alpha^{\prime}} \int_{\partial \Sigma} d \tau\left[i \xi^{\mu} \nabla_{\tau} \xi^{\nu} \mathscr{B}_{\mu \nu}+i \zeta^{i} \nabla_{\tau} \zeta^{j} F_{i j}+\rho \xi^{\mu} \tau_{\mu}+\bar{\rho} \xi^{\mu} \bar{\tau}_{\mu}\right. \\
&\left.+\zeta^{i} \zeta^{j}\left(\partial_{\sigma} X_{0}^{\rho} \partial_{i} f^{\mu} \partial_{j} f^{\nu} \nabla_{\mu} E_{\nu \rho}+i \partial_{\tau} Y_{0}^{k} \nabla_{i} \mathscr{F}_{j k}\right)\right] .
\end{aligned}
$$

It is understood that all the couplings are evaluated at $X=X_{0}$ and $Y=Y_{0}$. The background fields are required to satisfy the boundary conditions in (3.26) and therefore $N\left(Y_{0}\right)=0$. Here, $\mathscr{F}_{i j}$ is defined in (3.27) and

$$
\mathscr{H}_{\mu \nu \rho}=\partial_{\mu} \mathscr{B}_{\nu \rho}+\partial_{\rho} \mathscr{B}_{\mu \nu}+\partial_{\nu} \mathscr{B}_{\rho \mu} .
$$

The Riemann tensor is defined with respect to the Christoffel symbol $\Gamma_{\rho \sigma}^{\mu}$. 
To proceed to quantum calculations, it is convenient to change variables from $\xi^{\mu}$ to $\left(\xi^{A}, \xi^{A^{\prime}}\right)$ and from $\zeta^{i}$ to $\zeta^{I}=\left(\zeta^{0}, \zeta^{A^{\prime}}\right)$, with

$$
\xi^{A}=\tau_{\mu}{ }^{A} \xi^{\mu}, \quad \xi^{A^{\prime}}=E_{\mu}{ }^{A^{\prime}} \xi^{\mu}, \quad \zeta^{0}=t_{i}{ }^{0} \zeta^{i}, \quad \zeta^{A^{\prime}}=e_{i}{ }^{A^{\prime}} \zeta^{i} .
$$

In terms of these new variables, we have a simple propagator that is diagonalized. From (3.39), we find that the free part of the quadratic action is

$$
S_{\text {free }}=\frac{1}{4 \pi \alpha^{\prime}} \int_{\Sigma} d^{2} \sigma\left(\partial_{\alpha} \xi^{A^{\prime}} \partial^{\alpha} \xi^{A^{\prime}}+\rho \bar{\partial} \xi+\bar{\rho} \partial \bar{\xi}\right)
$$

with $\xi=\xi^{0}+\xi^{1}$ and $\bar{\xi}=\xi^{0}-\xi^{1}$. At the zeroth order in quantum fluctuations, $\xi^{A}, \xi^{A^{\prime}}, \rho$ and $\bar{\rho}$ satisfy the boundary conditions $(2.8)-(2.11)$, with

$$
\left.\partial_{\tau} \xi^{1}\right|_{\sigma=0}=\left.\partial_{\sigma} \xi^{0}\right|_{\sigma=0}=\left.\partial_{\sigma} \xi^{A^{\prime}}\right|_{\sigma=0}=\rho+\left.\bar{\rho}\right|_{\sigma=0}=0, \quad \partial_{\sigma} \xi^{1}+\left.i \partial_{\tau} \xi^{0}\right|_{\sigma=0}=0 .
$$

It follows from [27] that

$$
\left.\nabla_{\alpha} \xi^{\mu}\right|_{\sigma=0}=\tau_{A}^{\mu} \partial_{\alpha} \xi^{A}+E_{A^{\prime}}^{\mu}\left[\partial_{\alpha} \xi^{A^{\prime}}-\left(\Omega_{\nu}{ }^{0 A^{\prime}} \xi^{0}+\Omega_{\nu}{ }^{A^{\prime} B^{\prime}} \xi^{B^{\prime}}\right) \partial_{\alpha} X_{0}^{\nu}\right]
$$

There is ambiguity in identifying the covariant quantum fluctuation in $\left.(\rho-\bar{\rho})\right|_{\sigma=0}$ that one should integrate out on the boundary. It will prove to be useful to introduce the following decomposition of $\rho-\bar{\rho}$ on the boundary:

$$
\left.\frac{1}{2}(\rho-\bar{\rho})\right|_{\sigma=0}=r-\frac{i}{2} \omega_{A^{\prime}} \zeta^{A^{\prime}}-\frac{i}{2} \partial_{\tau}\left(\omega_{A^{\prime} B^{\prime}} \zeta^{A^{\prime}} \zeta^{B^{\prime}}\right)+O\left(\zeta^{3}\right) .
$$

We will show that $\omega_{A^{\prime}}$ and $\omega_{A^{\prime} B^{\prime}}$ are fixed by requiring that the one-loop effective action is gauge covariant. Using (3.34) that relates the bulk quantum field $\xi^{\mu}$ to the boundary quantum field $\zeta^{i}$, in terms of the new variables (3.41) which satisfy the free field boundary conditions (3.43), we read off the terms in (3.39) that are quadratic in quantum fields as

$$
\begin{aligned}
S_{2}= & -\frac{i}{2 \pi \alpha^{\prime}} \int_{\Sigma} d^{2} \sigma \xi^{I} \partial_{\tau} \xi^{J} \mathcal{W}_{I J} \\
& +\frac{1}{4 \pi \alpha^{\prime}} \int_{\partial \Sigma} d \tau\left(\zeta^{I} \zeta^{J} \mathcal{M}_{I J}+i \zeta^{I} \partial_{\tau} \zeta^{J} \mathscr{F}_{I J}+2 r \zeta^{I} t_{I}^{1}\right)
\end{aligned}
$$

where

$$
\mathcal{W}_{I J}=W_{I J}+i \Omega_{\mu I J} \partial_{\tau} X_{0}^{\mu}, \quad \mathcal{M}_{I J}=M_{I J}+M_{I J}^{\Omega}
$$

with

$$
\begin{aligned}
M_{i j} & =i \partial_{\tau} Y_{0}^{k}\left[\nabla_{(i} \mathscr{F}_{j) k}+\left(K^{\mu}{ }_{i j} \partial_{k} f^{\nu}+K_{k(i}^{\nu} \partial_{j)} f^{\mu}\right) \mathscr{B}_{\mu \nu}\right]+\frac{1}{2}(\lambda-\bar{\lambda}) K^{\mu}{ }_{i j} \tau_{\mu}{ }^{1}, \\
M_{00}^{\Omega} & =-i \Omega_{\tau}{ }^{0 A^{\prime}} \mathscr{F}_{0 A^{\prime}}, \quad W_{\mu \nu}=\frac{1}{2} \mathscr{H}_{\mu \nu \rho} \partial_{\sigma} X_{0}^{\rho}, \\
M_{0 A^{\prime}}^{\Omega} & =M_{A^{\prime} 0}^{\Omega}=-\frac{i}{2}\left(\Omega_{\tau}{ }^{0 B^{\prime}} \mathscr{F}_{A^{\prime} B^{\prime}}-\Omega_{\tau}{ }^{A^{\prime} B^{\prime}} \mathscr{F}_{0 B^{\prime}}+\omega_{A^{\prime}} t_{0}{ }^{1}\right) \\
M_{A^{\prime} B^{\prime}}^{\Omega} & =i\left[\Omega_{\tau\left(A^{\prime}\right.}{ }^{C^{\prime}} \mathscr{F}_{\left.B^{\prime}\right) C^{\prime}}-\partial_{\tau} Y_{0}^{i} t_{i}{ }^{1}\left(E^{\nu}{ }_{A^{\prime}} \Omega_{\nu}{ }^{0 B^{\prime}}-\omega_{A^{\prime} B^{\prime}}\right)-\omega_{\left(A^{\prime}\right.} t_{\left.B^{\prime}\right)}{ }^{1}\right] .
\end{aligned}
$$


The boundary conditions in (3.26) have been applied to derive (3.46). Note that $W_{I J}$ is $W_{\mu \nu}$ projected by $\tau^{\mu}$ and $E^{\mu} A^{\prime}$. Moreover, $M_{I J}, t_{I}{ }^{1}$ and $\mathscr{F}_{I J}$ are $M_{i j}, t_{i}{ }^{1}$ and $\mathscr{F}_{i j}$ projected by $t^{i}{ }_{0}$ and $e^{i} A^{\prime}$, respectively. We also defined $\Omega_{\tau}^{I J}=\partial_{\tau} Y_{0}^{k} \partial_{k} f^{\mu} \Omega_{\mu}{ }^{I J}$. Here, we only kept terms that will give rise to nontrivial contributions to the one-loop boundary effective action. To derive (3.46), we used the following identities:

$$
\begin{aligned}
& \left.\nabla_{\tau} \xi^{\mu}\right|_{\sigma=0}=\partial_{i} f^{\mu} \nabla_{\tau} \zeta^{i}+\zeta^{i} K^{\mu}{ }_{i j} \partial_{\tau} Y_{0}^{j}+O\left(\zeta^{2}\right), \\
& \tau_{0}^{\mu} \Omega_{\mu}{ }^{0 A^{\prime}}=0, \quad E^{\mu}{ }_{A^{\prime}} \Omega_{\mu}{ }^{0}{ }_{B^{\prime}}=E^{\mu}{ }_{B^{\prime}} \Omega_{\mu}{ }^{0}{ }_{A^{\prime}} .
\end{aligned}
$$

In the next subsection, we compute the one-loop effective action by integrating out the quantum fluctuations in the path integral associated with the action (3.46).

\subsection{Beta-functions for open string couplings}

From the free action (3.42), we read off the propagators, which are already given in (2.35) and (2.36),

$$
\begin{aligned}
\left\langle\xi^{A^{\prime}}(\tau, \sigma) \xi^{B^{\prime}}\left(\tau^{\prime}, \sigma^{\prime}\right)\right\rangle & =2 \pi \alpha^{\prime}\left[\Delta\left(\tau-\tau^{\prime}, \sigma-\sigma^{\prime}\right)+\Delta\left(\tau-\tau^{\prime}, \sigma+\sigma^{\prime}\right)\right], \\
\left\langle\rho(\tau, \sigma) \xi\left(\tau^{\prime}, \sigma^{\prime}\right)\right\rangle & =4 \pi \alpha^{\prime} \partial \Delta\left(\tau-\tau^{\prime}, \sigma-\sigma^{\prime}\right), \\
\left\langle\bar{\rho}(\tau, \sigma) \bar{\xi}\left(\tau^{\prime}, \sigma^{\prime}\right)\right\rangle & =4 \pi \alpha^{\prime} \bar{\partial} \Delta\left(\tau-\tau^{\prime}, \sigma-\sigma^{\prime}\right),
\end{aligned}
$$

where we defined

$$
\Delta(\tau, \sigma)=-\frac{1}{4 \pi} \ln \left(\tau^{2}+\sigma^{2}\right)=\int \frac{d \omega d k}{(2 \pi)^{2}} \frac{e^{i(\omega \tau+k \sigma)}}{\omega^{2}+k^{2}} .
$$

Since we are focusing on the boundary one-loop effective action, we are mostly interested in the boundary propagators with $\sigma=0$. Because $\rho, \bar{\rho}$ and $\xi^{A}$ only appear as boundary fields in the contributions we are interested in, we can set $\rho(\tau, 0)=-\bar{\rho}(\tau, 0) \sim r(\tau)$ in (3.49). It follows that the boundary-boundary propagators are

$$
\begin{aligned}
\left\langle\xi^{A^{\prime}}(\tau, 0) \xi^{B^{\prime}}\left(\tau^{\prime}, 0\right)\right\rangle & =4 \pi \alpha^{\prime} \Delta\left(\tau-\tau^{\prime}, 0\right), \\
\left\langle r(\tau) \xi^{0}\left(\tau^{\prime}, 0\right)\right\rangle & =-4 \pi i \alpha^{\prime} \partial_{\tau} \Delta\left(\tau-\tau^{\prime}, 0\right), \\
\left\langle r(\tau) \xi^{1}\left(\tau^{\prime}, 0\right)\right\rangle & =0 .
\end{aligned}
$$

Introducing the index $\mathcal{A}=(r, I)$, we write the boundary-boundary propagator as

$$
\Delta^{\mathcal{A B}}\left(\tau-\tau^{\prime}, 0\right)=2 \pi \alpha^{\prime}\left(\begin{array}{ccc}
0 & -i \partial_{\tau} & 0 \\
i \partial_{\tau} & 0 & 0 \\
0 & 0 & \delta^{A^{\prime} B^{\prime}}
\end{array}\right) \Delta\left(\tau-\tau^{\prime}\right)
$$

where we defined

$$
\Delta(\tau) \equiv 2 \Delta(\tau, 0)=\int \frac{d \omega}{2 \pi} \frac{e^{i \omega \tau}}{|\omega|}=-\frac{1}{\pi} \ln \left|\frac{\tau}{\tau_{\mathrm{IR}}}\right|,
$$

with $\tau_{\text {IR }}$ an infrared regulator. Moreover, using a sharp-cutoff regularization in the frequency space, we find

$$
\Delta(0)=\frac{1}{\pi} \log \left(\frac{\Lambda}{\mu}\right)
$$


with $\Lambda$ the ultraviolet cutoff and $\mu$ the infrared cutoff of the frequency. We introduced a prefactor 2 in the definition (3.53) of $\Delta(\tau)$, such that

$$
\int d \tau^{\prime \prime} \Delta^{-1}\left(\tau-\tau^{\prime \prime}\right) \Delta\left(\tau^{\prime \prime}-\tau^{\prime}\right)=\delta\left(\tau-\tau^{\prime}\right), \quad \Delta^{-1}\left(\tau, \tau^{\prime}\right) \equiv \partial_{\tau} \partial_{\tau^{\prime}} \Delta\left(\tau-\tau^{\prime}\right) .
$$

From the quadratic action (3.46) that collects terms relevant to the one-loop boundary effective action, we read off the following Feynman rules for different vertices:

$$
\begin{aligned}
V_{\mathcal{A B}}^{\mathcal{W}}\left(\tau, \sigma ; \tau^{\prime}, \sigma^{\prime}\right) & =\frac{i}{\pi \alpha^{\prime}}\left(\begin{array}{cc}
0 & 0 \\
0 & \mathcal{W}_{I J}(\tau, \sigma) \partial_{\tau}
\end{array}\right) \delta\left(\tau-\tau^{\prime}\right) \delta\left(\sigma-\sigma^{\prime}\right) \\
V_{\mathcal{A B}}^{\mathscr{F}}\left(\tau, \tau^{\prime}\right) & =-\frac{1}{2 \pi \alpha^{\prime}}\left(\begin{array}{cc}
0 & t_{J}^{1} \\
t_{I}{ }^{1} & i \mathscr{F}_{I J}(\tau) \partial_{\tau}
\end{array}\right) \delta\left(\tau-\tau^{\prime}\right) \\
V_{\mathcal{A B}}^{\mathcal{M}}\left(\tau, \tau^{\prime}\right) & =-\frac{1}{2 \pi \alpha^{\prime}}\left(\begin{array}{cc}
0 & 0 \\
0 & \mathcal{M}_{I J}
\end{array}\right) \delta\left(\tau-\tau^{\prime}\right)
\end{aligned}
$$

The loop calculation is very similar to the procedure discussed in $[2,30]$. All the one-loop diagrams are collected below,
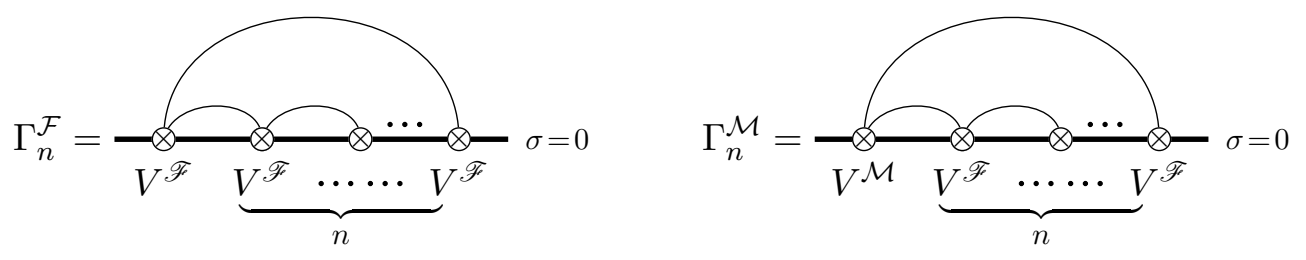

and

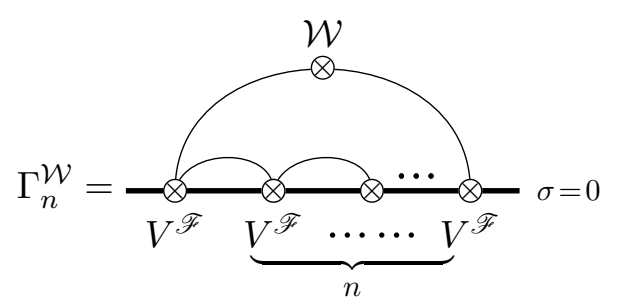

where the thick horizontal lines represent the boundary line $\sigma=0$, and the thin curved lines represent the propagator in (3.49). In particular, a propagator connecting two boundary vertices is given in (3.52). All the boundary vertices reside on the $\sigma=0$ boundary line in the diagrams. Similarly to the case of relativistic string theory in [30], the above diagrams in the sum are only nonzero when $n$ is even. A careful analysis shows that the sums of the Feynman diagrams in (3.57) give

$$
\sum_{n=0}^{\infty} \Gamma_{n}^{\mathscr{F}}=-\frac{i}{2} \Delta(0) \int d \tau J^{r I}(\tau) \partial_{\tau} t_{I}^{1}(\tau)+\text { finite }
$$

and

$$
\sum_{n=0}^{\infty} \Gamma_{n}^{\mathcal{M}}=-\frac{1}{2} \Delta(0) \int d \tau J^{I J}(\tau) \mathcal{M}_{I J}(\tau)+\text { finite }
$$


With the definition $\mathcal{A}=\{r, I\}$, we have

$$
J^{\mathcal{A B}}=\left(\begin{array}{cc}
J^{r r} & J^{r J} \\
J^{I r} & J^{I J}
\end{array}\right)
$$

which is defined to be the inverse of

$$
J_{\mathcal{A B}}=G_{\mathcal{A B}}-\mathscr{F}_{\mathcal{A C}} G^{\mathcal{C D}} \mathscr{F}_{\mathcal{D B}}
$$

where

$$
G_{\mathcal{A B}}=\left(\begin{array}{ccc}
0 & 1 & 0 \\
1 & 0 & 0 \\
0 & 0 & \delta^{A^{\prime} B^{\prime}}
\end{array}\right), \quad \mathscr{F}_{\mathcal{A B}}=\left(\begin{array}{cc}
0 & t_{J}^{1} \\
-t_{I}{ }^{1} & \mathscr{F}_{I J}
\end{array}\right)
$$

and $G^{\mathcal{A B}}$ is the inverse of $G_{\mathcal{A B}}$. In our calculation that leads to (3.59), the total derivatives in (3.59) are dropped. This is consistent with that the one-loop effective action is defined up to total derivative terms. However, the full expression of $J^{r I}$ in (3.59) is needed for maintaining the gauge invariance when the beta-functions are concerned. See related discussions for the closed string beta-functions in [27].

Similarly, the sum of the Feynmann diagrams in (3.58) gives

$$
\sum_{n=0}^{\infty} \Gamma_{n}^{\mathcal{W}}=-\frac{1}{2} \Delta(0) \int d \tau J^{I \mathcal{A}}(\tau) \mathcal{W}_{I J}(\tau, 0) \mathscr{F}^{J}{ }_{\mathcal{A}}(\tau)+\text { finite }
$$

where, by definition, $\mathscr{F}_{\mathcal{B}}^{J}=G^{J \mathcal{A}} \mathscr{F}_{\mathcal{A B}}$. We have taken a Taylor expansion of $\mathcal{W}_{I J}(\tau, \sigma)$ around $\sigma=0$ and only kept the zeroth order term $\mathcal{W}_{I J}(\tau, 0)$ in this calculation.

Summing (3.59), (3.60) and (3.64), and using (3.29) to convert the indices 0 and $A^{\prime}$ back to $i$, we find the one-loop boundary effective action,

$$
\begin{aligned}
S_{\text {1-loop }}^{\text {bdry }}= & -\frac{1}{2} \Delta(0) \int_{\partial \Sigma} d \tau\left[i J^{r i} \nabla_{\tau} t_{i}{ }^{1}+J^{i j} M_{i j}-\frac{1}{2} J^{i a} \mathscr{F}_{a}{ }^{j} \partial_{i} f^{\mu} \partial_{j} f^{\nu} \mathscr{H}_{\mu \nu \rho} \partial_{\sigma} X_{0}^{\rho}\right] \\
& -\frac{i}{2} \Delta(0) \int_{\partial \Sigma} d \tau\left[J^{A^{\prime} I}\left(\Omega_{\tau}{ }^{0 A^{\prime}}-\omega_{A^{\prime}}\right) t_{I}{ }^{1}-J^{A^{\prime} B^{\prime}}\left(E^{\mu}{ }_{A^{\prime}} \Omega_{\mu}{ }^{0 B^{\prime}}-\omega_{A^{\prime} B^{\prime}}\right) t_{\tau}{ }^{1}\right],
\end{aligned}
$$

where $\mathscr{H}_{\mu \nu \rho}$ is evaluated at $\sigma=0$ and $M_{i j}$ is given in (3.46); $J^{a b}$ with $a=(r, i)$ is defined to be the inverse of

$$
J_{a b}=G_{a b}-\mathscr{F}_{a c} \mathscr{F}^{c}{ }_{b} .
$$

Here,

$$
G_{a b}=\left(\begin{array}{cc}
0 & t_{j}{ }^{0} \\
t_{i}{ }^{0} & e_{i j}
\end{array}\right), \quad \mathscr{F}_{a b}=\left(\begin{array}{cc}
0 & t_{j}{ }^{1} \\
-t_{i}{ }^{1} & \mathscr{F}_{i j}
\end{array}\right),
$$

and we defined $e_{i j}=e_{i}{ }^{A^{\prime}} e_{j}{ }^{B^{\prime}}$, with $e_{i}{ }^{A^{\prime}}$ defined in (3.27). The lower indices can be raised by $G^{a b}$, which is the inverse of $G_{a b}$,

$$
G^{a b}=\left(\begin{array}{cc}
0 & t^{j} \\
t^{i}{ }_{0} & e^{i j}
\end{array}\right)
$$


where $e^{i j}=e^{i} A^{\prime} e^{j} B^{\prime}$, with $e^{i} A^{\prime}$ defined by (3.28). The gauge covariance of $S_{1-\text { loop }}$ in (3.65) requires in $(3.45)$

$$
\omega_{A^{\prime}}=\Omega_{\tau}{ }^{0 A^{\prime}}, \quad \omega_{A^{\prime} B^{\prime}}=E^{\mu}{ }_{A^{\prime}} \Omega_{\mu}{ }^{0 B^{\prime}} .
$$

Applying the boundary conditions (3.26), we rewrite (3.65) as

$$
S_{1 \text {-loop }}=-\frac{1}{2 \alpha^{\prime}} \Delta(0) \int_{\partial \Sigma} d \tau\left[\frac{1}{2}(\lambda-\bar{\lambda}) \beta(N)+i \partial_{\tau} Y^{k} \beta_{k}(A)\right],
$$

where

$$
\begin{array}{r}
\beta(N)=\alpha^{\prime}\left(J^{i j} K^{\mu}{ }_{i j} \tau_{\mu}{ }^{1}+\frac{1}{2} J^{i a} \mathscr{F}_{a}{ }^{j} \partial_{i} f^{\mu} \partial_{j} f^{\nu} \mathscr{H}_{\mu \nu \rho} E^{\rho}{ }_{A^{\prime}} t_{A^{\prime}}{ }^{1}\right)+O\left(\alpha^{\prime}\right)^{2}, \\
\beta_{k}(A)=\alpha^{\prime}\left\{J^{r i} \nabla_{k} t_{i}{ }^{1}-\frac{1}{2} J^{i a} \mathscr{F}_{a}{ }^{j} \partial_{i} f^{\mu} \partial_{j} f^{\nu} \mathscr{H}_{\mu \nu \rho}\left(\epsilon^{A}{ }_{B} \tau^{\rho}{ }_{A} t_{k}{ }^{B}-E^{\rho}{ }_{A^{\prime}} \mathscr{F}_{A^{\prime} k}\right)\right. \\
\left.+J^{i j}\left[\nabla_{i} \mathscr{F}_{j k}+\left(K^{\mu}{ }_{i j} \partial_{k} f^{\nu}+K^{\nu}{ }_{k i} \partial_{j} f^{\mu}\right) \mathscr{B}_{\mu \nu}\right]\right\}+O\left(\alpha^{\prime}\right)^{2}
\end{array}
$$

are the beta-functions for the couplings $N$ and $A_{k}$ in (3.24).

\subsection{Contribution from the dilaton}

Finally, we discuss the contribution from including a dilaton field in the sigma model action, which is only at the classical level when the lowest order of $\alpha^{\prime}$ is concerned [2]. On a curved worldsheet equipped with a metric $h_{\alpha \beta}, \alpha, \beta=1,2$, we have the dilaton term in the action,

$$
S_{\Phi}=\frac{1}{4 \pi} \int d^{2} \sigma \sqrt{h} R[h] \Phi[x]
$$

where $R[h]$ is the worldsheet Ricci scalar and $\Phi[x]$ is the dilaton field. This dilaton term vanishes identically in the flat worldsheet limit, but it contributes nontrivially to the boundary stress energy tensor

$$
\begin{aligned}
T_{\Phi} & =-\left.\partial_{\sigma} \Phi\right|_{\sigma=0} \\
& =\frac{1}{2}(\lambda-\bar{\lambda}) t_{A^{\prime}}{ }^{1} E^{\mu}{ }_{A^{\prime}} \partial_{\mu} \Phi-i \partial_{\tau} Y^{k} \partial_{\mu} \Phi\left(\epsilon_{B}^{A} \tau_{A}^{\mu} t_{k}{ }^{B}-E_{A^{\prime}}^{\mu} \mathscr{F}_{A^{\prime} k}\right) .
\end{aligned}
$$

The trace of the boundary stress energy tensor is related to the boundary beta-functions $\beta(N)$ and $\beta_{k}(A)$ by

$$
T=-\frac{1}{\alpha^{\prime}}\left[\frac{1}{2}(\lambda-\bar{\lambda}) \beta(N)+i \partial_{\tau} Y^{k} \beta_{k}(A)\right] .
$$

Therefore, the beta-functions in (3.71) are modified to be

$$
\begin{aligned}
& \beta(N)=\alpha^{\prime}\left(J^{i j} K^{\mu}{ }_{i j} \tau_{\mu}{ }^{1}+\Theta_{\rho} E^{\rho}{ }_{A^{\prime}} t_{A^{\prime}}{ }^{1}\right)+O\left(\alpha^{\prime}\right)^{2}, \\
& \beta_{k}(A)=\alpha^{\prime}\left\{J^{r i} \nabla_{k} t_{i}{ }^{1}+J^{i j}\left[\nabla_{i} \mathscr{F}_{j k}+\left(K^{\mu}{ }_{i j} \partial_{k} f^{\nu}+K^{\nu}{ }_{k i} \partial_{j} f^{\mu}\right) \mathscr{B}_{\mu \nu}\right]\right. \\
&\left.-\Theta_{\rho}\left(\epsilon^{A}{ }_{B} \tau^{\rho}{ }_{A} t_{k}{ }^{B}-E^{\rho}{ }_{A^{\prime}} \mathscr{F}_{A^{\prime} k}\right)\right\}+O\left(\alpha^{\prime}\right)^{2},
\end{aligned}
$$


where

$$
\Theta_{\rho}=\frac{1}{2} J^{i a} \mathscr{F}_{a}^{j} \partial_{i} f^{\mu} \partial_{j} f^{\nu} \mathscr{H}_{\mu \nu \rho}-\partial_{\rho} \Phi .
$$

Setting the beta-functions $\beta(N)$ and $\beta_{k}(A)$ to zero gives rise to the equations of motion that determine the backgrounds on which nonrelativistic open string theory can consistently propagate. Note that the beta-functions of the closed string background fields are not affected at this lowest order in $\alpha^{\prime}$.

\section{Nonrelativistic Dirac-Born-Infeld effective theory}

In this section, we introduce a DBI-like action that is invariant under the Bargmann symmetry, which describes the effective field theory living on the D-brane. We will show that the classical equations of this DBI action are equivalent to the vanishing one-loop beta-functions in (3.75).

\subsection{Galilean DBI action from a nonrelativistic limit}

To determine the brane action, we start with the relativistic worldvolume DBI action on a $\mathrm{D}(d-2)$-brane,

$$
\widehat{S}_{\text {brane }}=T_{d-2} \int d^{d-1} Y e^{-\widehat{\Phi}} \sqrt{-\operatorname{det}\left(\widehat{g}_{i j}+\widehat{\mathcal{F}}_{i j}\right)},
$$

where $\widehat{g}_{i j}=\partial_{i} f^{\mu} \partial_{j} f^{\nu} \widehat{G}_{\mu \nu}, \widehat{\mathcal{F}}_{i j}=\partial_{i} f^{\mu} \partial_{j} f^{\nu} \widehat{B}_{\mu \nu}+F_{i j}$. Here, $f^{\mu}$ is the embedding function that describes how the D-brane is embedded in a $d$-dimensional spacetime. Consider the following expansions with respect to a large parameter $c[12]:^{18}$

$$
\widehat{G}_{\mu \nu}=c^{2} \tau_{\mu \nu}+H_{\mu \nu}, \quad \widehat{B}_{\mu \nu}=-c^{2} \tau_{\mu}{ }^{A} \tau_{\nu}{ }^{B} \epsilon_{A B}+B_{\mu \nu}, \quad \widehat{\Phi}=\Phi+\ln |c| .
$$

Then, (4.1) becomes

$$
\widehat{S}_{\text {brane }}=T_{d-2} \int d^{d-1} Y e^{-\Phi} \sqrt{-c^{-2} \operatorname{det}\left(h_{i j}+\mathcal{F}_{i j}-c^{2} \bar{t}_{i} t_{j}\right)}
$$

where $t_{i}{ }^{A}=\tau_{\mu}{ }^{A} \partial_{i} f^{\mu}, h_{i j}=\partial_{i} f^{\mu} \partial_{j} f^{\nu} H_{\mu \nu}$, and $\mathcal{F}_{i j}=\partial_{i} f^{\mu} \partial_{j} f^{\nu} B_{i j}+F_{i j}$. We also defined $t_{i}=t_{i}{ }^{0}+t_{i}{ }^{1}$ and $\bar{t}_{i}=t_{i}{ }^{0}-t_{i}{ }^{1}$. Using the identities ${ }^{19}$

$$
\begin{aligned}
\operatorname{det}\left(O_{i j}-c^{2} \bar{t}_{i} t_{j}\right) & =\left(1-c^{2} t_{m} O^{m n} \bar{t}_{n}\right) \operatorname{det} O_{k \ell}, \\
\operatorname{det}\left(\begin{array}{cc}
0 & t_{j} \\
\bar{t}_{i} & O_{i j}
\end{array}\right) & =\left(-t_{m} O^{m n} \bar{t}_{n}\right) \operatorname{det} O_{k \ell},
\end{aligned}
$$

with $O^{i j}$ the inverse of $O_{i j} \equiv h_{i j}+\mathcal{F}_{i j}$, we find

$$
\lim _{c \rightarrow \infty} \widehat{S}_{\text {brane }}=S_{\text {brane }}
$$

\footnotetext{
${ }^{18}$ Note that this is different from the $\mathrm{D} p$-brane limit considered in $[4,9,11,31,32]$, which involves the $\mathrm{RR}$ charges. In the D $p$-brane limit, there are no light strings left.

${ }^{19}$ These expressions are non-singular in the limit $m_{i}{ }^{A} \rightarrow 0$ and $\mathcal{F}_{i j} \rightarrow 0$ [12].
} 
where $^{20}$

$$
S_{\text {brane }}=T_{d-2} \int d^{d-1} Y e=\sqrt{-\operatorname{det}\left(\begin{array}{cc}
0 & t_{j} \\
\bar{t}_{i} & h_{i j}+\mathcal{F}_{i j}
\end{array}\right)} .
$$

The action (4.6) is invariant under the string Newton-Cartan gauge symmetries (3.2), supplemented with the transformation of $A_{i}$ in (3.17). This action is also invariant under the Stückelberg transformations (3.7), under which the gauge field $A_{i}$ remains invariant. The invariance under the string Newton-Cartan gauge symmetry and the Stückelberg transformations can be shown by using the identity

$$
\operatorname{det}\left(\begin{array}{cc}
0 & t_{j} \\
\bar{t}_{i} & O_{i j}+a_{i} t_{j}+b_{j} \bar{t}_{i}
\end{array}\right)=\operatorname{det}\left(\begin{array}{cc}
0 & t_{j} \\
\bar{t}_{i} & O_{i j}
\end{array}\right),
$$

for arbitrary $O_{i j}, a_{i}$ and $b_{i}$.

\subsection{Equations of motion}

To derive the equations of motion of the brane action (4.6), we start with performing a field redefinition using (3.7) with $C_{\mu}{ }^{A}=m_{\mu}{ }^{A}$, and rewrite (4.6) as

$$
S_{\text {brane }}=T_{d-2} \int d^{d-1} Y e^{-\Phi} \sqrt{-\operatorname{det}\left(G_{a b}+\mathscr{F}_{a b}\right)},
$$

with $G_{a b}$ and $\mathscr{F}_{a b}$ defined in (3.67). To extract the appropriate equations of motion from (4.8) to compare with the vanishing beta-functions in (3.75), it is useful to use the adapted coordinates introduced in the "broken phase" in section 3.2, where the string Newton-Cartan symmetry is broken to the Bargmann symmetry, with $X^{\mu}=\left(y, Y^{i}\right)$ and

$$
f^{y}=y_{0}+N, \quad f^{i}=Y^{i}
$$

Varying the action (4.8) with respect to $N$ and $A_{i}$, we find

$$
\begin{aligned}
\delta S_{\text {brane }}=-T_{d-2} \int d^{d-1} Y e^{-\Phi} \sqrt{-\operatorname{det}(G+\mathscr{F})} & \\
& \times\left\{\mathcal{E}_{a}^{(A)} J^{a k} \delta A_{k}+\left[\mathcal{E}^{(N)}+\mathcal{E}_{a}^{(A)}\left(J^{a r}+J^{a i} B_{i y}\right)\right] \delta N\right\},
\end{aligned}
$$

where $a=(r, i)$ and

$$
\begin{aligned}
& \mathcal{E}_{r}^{(A)}=-J^{i j} \nabla_{i} t_{j}{ }^{1}-t_{A^{\prime}}{ }^{1} e^{i}{ }_{A^{\prime}} \theta_{i}, \\
& \mathcal{E}_{k}^{(A)}=J^{i r} \nabla_{i} t_{k}{ }^{1}+J^{i j}\left[\nabla_{i} \mathscr{F}_{j k}+\mathscr{B}_{\mu \nu}\left(K^{\mu}{ }_{i j} \partial_{k} f^{\nu}+K^{\nu}{ }_{i k} \partial_{j} f^{\mu}\right)\right]+\theta_{i} \mathscr{F}^{i}{ }_{k}, \\
& \mathcal{E}^{(N)}=\frac{1}{2} J^{a i} \mathscr{F}_{a}{ }^{j} \partial_{i} f^{\mu} \partial_{j} f^{\nu} \mathscr{H}_{\mu \nu y}-\partial_{y} \Phi=\Theta_{y} .
\end{aligned}
$$

Here, the definition of $\Theta_{y}$ matches the one in (3.76). We also defined

$$
\theta_{k}=\frac{1}{2} J^{a i} \mathscr{F}_{a}^{j} \partial_{i} f^{\mu} \partial_{j} f^{\nu} \partial_{k} f^{\rho} \mathscr{H}_{\mu \nu \rho}-\partial_{k} f^{\mu} \partial_{\mu} \Phi .
$$

\footnotetext{
${ }^{20} \mathrm{~A}$ related worldvolume action for D-branes has been considered in [33, 34], where the embedding spacetime is taken to be torsional Newton-Cartan spacetime extended with a periodic space direction [26, 35].
} 
By the definition $t_{i}{ }^{1}=\partial_{i} f^{\mu} \tau_{\mu}{ }^{1}$, we have $t_{i}{ }^{1}=\tau_{i}{ }^{1}+\partial_{i} N$. Comparing (4.12) with (3.76), it follows that

$$
\theta_{i}=\Theta_{i}+\Theta_{y} \partial_{i} N
$$

Requiring $\delta S_{\text {brane }}=0$ gives

$$
\mathcal{E}_{a}^{(A)} J^{a i}=0, \quad \mathcal{E}^{(N)}+\mathcal{E}_{a}^{(A)} J^{a r}=0 .
$$

Using the definitions $J^{a c} J_{c b}=\delta_{b}^{a}$ and $J_{a b}=G_{a b}-\mathscr{F}_{a c} \mathscr{F}_{b}^{c}$ in (3.66), we find that (4.14) is equivalent to

$$
\begin{aligned}
& \mathcal{E}_{r}^{(A)}=\left(\mathcal{E}_{a}^{(A)} J^{a i}\right) J_{i r}+\left(\mathcal{E}_{a}^{(A)} J^{a r}\right) J_{r r}=-\mathcal{E}^{(N)} t_{A^{\prime}}{ }^{1} t_{A^{\prime}}{ }^{1}, \\
& \mathcal{E}_{k}^{(A)}=\left(\mathcal{E}_{a}^{(A)} J^{a i}\right) J_{i k}+\left(\mathcal{E}_{a}^{(A)} J^{a r}\right) J_{r k}=-\mathcal{E}^{(N)}\left(t_{k}{ }^{0}-t_{0}{ }^{1} t_{k}{ }^{1}-t_{A^{\prime}}{ }^{1} \mathscr{F}_{A^{\prime} k}\right) .
\end{aligned}
$$

Plugging (4.11) into (4.15), we find

$$
\begin{aligned}
0=J^{i j} \nabla_{i} t_{j}{ }^{1} & +t_{A^{\prime}}{ }^{1}\left[e^{i}{ }_{A^{\prime}}\left(\Theta_{i}+\Theta_{y} \partial_{i} N\right)-t_{A^{\prime}}{ }^{1} \Theta_{y}\right] \\
0=J^{i r} \nabla_{i} t_{k}{ }^{1} & +J^{i j}\left[\nabla_{i} \mathscr{F}_{j k}+\mathscr{B}_{\mu \nu}\left(K^{\mu}{ }_{i j} \partial_{k} f^{\nu}+K^{\nu}{ }_{i k} \partial_{j} f^{\mu}\right)\right] \\
& +\left(\Theta_{i}+\Theta_{y} \partial_{i} N\right) \mathscr{F}_{k}{ }_{k}+\Theta_{y}\left(t_{k}{ }^{0}-t_{0}{ }^{1} t_{i}{ }^{1}-t_{A^{\prime}}{ }^{1} \mathscr{F}_{A^{\prime} k}\right) .
\end{aligned}
$$

In order to show that the equations of motion in (4.16) match with the vanishing beta-functions in (3.75), we need to use the identities in (3.21) and (3.37), with

$$
\tau^{i}{ }_{1}=0, \quad \tau^{y}{ }_{0}=-\tau_{0}^{i} \tau_{i}{ }^{1}, \quad E^{y} A^{\prime}=-E^{i}{ }_{A^{\prime}} \tau_{i}{ }^{1}, \quad \tau_{\mu}{ }^{1} K^{\mu}{ }_{i j}=\nabla_{i} t_{j}{ }^{1}=\nabla_{j} t_{i}{ }^{1} .
$$

Moreover, using the prescriptions in (3.21) and (3.22), together with the invertibility condition in (3.28), we find

$$
\tau_{0}^{i}=t_{0}^{i}, \quad E_{A^{\prime}}^{i}=e_{A^{\prime}}^{i}
$$

Applying (4.17) and (4.18) to (4.16), we find

$$
\begin{aligned}
0=J^{i j} K^{\mu}{ }_{i j} \tau_{\mu}{ }^{1}+t_{A^{\prime}}{ }^{1} E^{\rho}{ }_{A^{\prime}} \Theta_{\rho} \\
0=J^{i r} \nabla_{k} t_{i}{ }^{1}+J^{i j}\left[\nabla_{i} \mathscr{F}_{j k}+\mathscr{B}_{\mu \nu}\left(K^{\mu}{ }_{i j} \partial_{k} f^{\nu}+K^{\nu}{ }_{i k} \partial_{j} f^{\mu}\right)\right] \\
-\Theta_{\rho}\left(\epsilon^{A}{ }_{B} \tau^{\rho}{ }_{A} t_{k}{ }^{B}-E^{\rho}{ }_{A^{\prime}} \mathscr{F}_{A^{\prime} k}\right) .
\end{aligned}
$$

These are precisely the same equations from setting the beta-functions in (3.75) to zero.

\subsection{Galilean electrodynamics on a Newton-Cartan background}

It is interesting to consider the worldvolume action (4.6) in a simple case where we assume that $y$ is an isometry direction and take the following specifications on the boundary:

$$
\tau_{i}{ }^{1}=m_{i}{ }^{1}=m_{y}{ }^{1}=m_{y}{ }^{0}=B_{\mu \nu}=\Phi=0 .
$$

We therefore consider a zero B-field and dilaton background. Then, the worldvolume action (4.6) reduces to

$$
S_{\text {brane }}=T_{d-2} \int d^{d-1} Y \sqrt{-\operatorname{det}\left(\mathscr{G}_{a b}+\mathcal{F}_{a b}\right)},
$$


where

$$
\mathscr{G}_{a b}=\left(\begin{array}{cc}
0 & \tau_{j}^{0} \\
\tau_{i}^{0} & H_{i j}
\end{array}\right), \quad \mathcal{F}_{a b}=\left(\begin{array}{cc}
0 & \partial_{j} N \\
-\partial_{i} N & F_{i j}
\end{array}\right),
$$

and $H_{i j}=E_{i j}-\left(m_{i}{ }^{0} \tau_{j}{ }^{0}+m_{j}{ }^{0} \tau_{i}{ }^{0}\right)$, with $E_{i j}=E_{i}{ }^{A^{\prime}} E_{j}{ }^{B^{\prime}}$. The action in (4.21) can be further rewritten as

$$
S_{\text {brane }}=T_{d-2} \int d^{d-1} Y \sqrt{-\mathscr{G}} \sqrt{\operatorname{det}\left(\delta_{b}^{a}+\mathscr{G}^{a c} \mathcal{F}_{c b}\right)},
$$

where $\mathscr{G}$ is the determinant of $\mathscr{G}_{a b}$ and $\mathscr{G}^{a b}$ is the inverse of $\mathscr{G}_{a b}$, with

$$
\mathscr{G}=-\left(\tau_{k}^{0} H^{k \ell} \tau_{\ell}^{0}\right) \operatorname{det} H_{i j}, \quad \mathscr{G}^{a b}=\left(\begin{array}{cc}
2 \phi & T^{j} \\
T_{0}^{i} & E^{i j}
\end{array}\right),
$$

where $E^{i j}=E^{i}{ }^{\prime} E^{j} B^{\prime}$. Note that $\mathscr{G}$ is independent of $m_{\mu}{ }^{0}$. We also defined

$$
\phi=m_{i}{ }^{0} \tau_{0}^{i}+\frac{1}{2} m_{i}{ }^{0} E^{i j} m_{j}{ }^{0}, \quad T^{i}=\tau_{0}^{i}+E^{i k} m_{k}{ }^{0} .
$$

Here, $\phi$ is related to the Newton potential. At the quadratic order in fields, (4.23) gives Galilean Electrodynamics on a Newton-Cartan background,

$$
\begin{aligned}
S_{\mathrm{GED}} & =\frac{1}{4 g^{2}} \int d^{25} Y \sqrt{-\mathscr{G}} \mathscr{G}^{a b} \mathcal{F}_{b c} \mathscr{G}^{c d} \mathcal{F}_{d a} \\
& =\frac{1}{g^{2}} \int d^{25} Y \sqrt{-\mathscr{G}}\left[\frac{1}{2}\left(T^{i} T^{j}-2 \phi E^{i j}\right) \nabla_{i} N \nabla_{j} N+E^{i k}\left(T^{\ell} \nabla_{i} N-\frac{1}{4} E^{j \ell} F_{i j}\right) F_{k \ell}\right] .
\end{aligned}
$$

Alternatively, we can also start with the equivalent worldvolume action (4.8), which now reduces to

$$
S_{\text {brane }}=T_{d-2} \int d^{d-1} Y \sqrt{-\operatorname{det}\left(G_{a b}+\mathscr{F}_{a b}\right)},
$$

where

$$
G_{a b}=\left(\begin{array}{cc}
0 & \tau_{j}^{0} \\
\tau_{i}^{0} & E_{i j}
\end{array}\right), \quad \mathscr{F}_{a b}=\left(\begin{array}{cc}
0 & \partial_{j} N \\
-\partial_{i} N & \mathscr{F}_{i j}
\end{array}\right),
$$

and $\mathscr{F}_{i j}=F_{i j}+m_{i} \partial_{j} N-m_{j} \partial_{i} N$, or, equivalently,

$$
\mathscr{F}_{i j}=\partial_{i} \mathscr{A}_{j}-\partial_{j} \mathscr{A}_{i}+N\left(\partial_{i} m_{j}{ }^{0}-\partial_{j} m_{i}{ }^{0}\right), \quad \mathscr{A}_{i}=A_{i}-m_{i}{ }^{0} N .
$$

At the quadratic order in fields, (4.27) gives an alternative form of Galilean Electrodynamics on a Newton-Cartan background,

$$
\begin{aligned}
S_{\mathrm{GED}} & =\frac{1}{4 g^{2}} \int d^{25} Y \sqrt{-G} G^{a b} \mathscr{F}_{b c} G^{c d} \mathscr{F}_{d a} \\
& =\frac{1}{g^{2}} \int d^{25} Y \sqrt{-G}\left[\frac{1}{2} \tau^{i}{ }_{0} \tau^{j}{ }_{0} \nabla_{i} N \nabla_{j} N+E^{i k}\left(\tau^{\ell}{ }_{0} \nabla_{i} N-\frac{1}{4} E^{j \ell} \mathscr{F}_{i j}\right) \mathscr{F}_{k \ell}\right],
\end{aligned}
$$

which is equivalent to (4.26). Here, $G$ is the determinant of $G_{a b}$ and $G=\mathscr{G}$.

The equivalent actions (4.26) and (4.30) coincide with the actions considered in [20]. In the flat spacetime limit, with $\tau_{i}{ }^{0}=\delta_{i}^{0}, E_{i}{ }^{A^{\prime}}=\delta_{i}^{A^{\prime}}$ and $m_{i}{ }^{0}=0$, both (4.26) and (4.30) reduce to the action of Galilean Electrodynamics in (2.47). 


\section{Conclusions}

In this paper, we have studied the Dirichlet nonlinear sigma model that describes nonrelativistic open strings ending on D-branes in the string Newton-Cartan geometry, KalbRamond and dilaton closed string background. Having a nonrelativistic open string spectrum requires the D-branes to be transverse to the longitudinal spatial direction in the string Newton-Cartan geometry background. We have computed the beta-functions for the open string vertex operators on a single $\mathrm{D}(d-2)$-brane, transverse to the longitudinal spatial direction. Self-consistency of nonrelativistic open string theory requires setting these beta-functions to zero, which gives rise to the equations of motion that govern the dynamics of the D-brane. We also showed that the same set of equations of motion arise from an action principle in (4.6), which is the worldvolume nonrelativistic DBI action of the D-brane. In a companion paper [25], we will consider T-duals of nonrelativistic open string theory.

At leading order in $\alpha^{\prime}$ and in the flat closed string background, the DBI action in (4.6) gives rise to Galilean Electrodynamics (GED). GED is a non-dynamical U(1) gauge theory that is invariant under a Galilean boost transformation, and has been studied at the classical level in [18-20, 36]. In [37], the one-loop beta-functions of Galilean electrodynamics coupled to a Schrödinger scalar in $2+1$ dimensions are computed, where the renormalization of the dynamical Schrödinger scalar receives highly nontrivial contributions from interactions with the non-dynamical gauge sector. There is an extra scalar in addition to the $\mathrm{U}(1)$ gauge field in GED, which finds a natural interpretation in nonrelativistic open string theory as the Nambu-Goldstone boson from spontaneously breaking the string Newton-Cartan symmetry algebra to the Bargmann symmetry algebra.

We also considered the low energy effective action on $n$ coincident D-branes, where our worldsheet analysis has led to a novel nonrelativistic $\mathrm{U}(n)$ Yang-Mills theory. Finally, we have shown how to incorporate open string winding modes and demonstrated that in spite of the nonlocal features of wound open strings that the nontrivial dynamics and spectrum of open strings is neatly captured by a gauge theory in one higher dimension, and interpreted the additional dimension as conjugate to winding number.

\section{Acknowledgments}

We would like to thank the organizers and participants at the online seminar series on "Non-Lorentzian Geometries: Non-Relativistic String Theory" (June 2020) for stimulating discussions, where results in this paper were first presented. This research is supported in part by Perimeter Institute for Theoretical Physics. Research at Perimeter Institute is supported in part by the Government of Canada through the Department of Innovation, Science and Economic Development Canada and by the Province of Ontario through the Ministry of Colleges and Universities.

Open Access. This article is distributed under the terms of the Creative Commons Attribution License (CC-BY 4.0), which permits any use, distribution and reproduction in any medium, provided the original author(s) and source are credited. 


\section{References}

[1] D.H. Friedan, Nonlinear models in $2+\epsilon$ dimensions, Annals Phys. 163 (1985) 318 [INSPIRE].

[2] R.G. Leigh, Dirac-Born-Infeld Action from Dirichlet Sigma Model, Mod. Phys. Lett. A 4 (1989) 2767 [INSPIRE].

[3] C.G. Callan Jr., C. Lovelace, C.R. Nappi and S.A. Yost, String Loop Corrections to $\beta$-functions, Nucl. Phys. B 288 (1987) 525 [InSPIRE].

[4] J. Gomis and H. Ooguri, Nonrelativistic closed string theory, J. Math. Phys. 42 (2001) 3127 [hep-th/0009181] [INSPIRE].

[5] I.R. Klebanov and J.M. Maldacena, $(1+1)$-dimensional NCOS and its $\mathrm{U}(N)$ gauge theory dual, Int. J. Mod. Phys. A 16 (2001) 922 [Adv. Theor. Math. Phys. 4 (2000) 283] [hep-th/0006085] [INSPIRE].

[6] U.H. Danielsson, A. Guijosa and M. Kruczenski, IIA/B, wound and wrapped, JHEP 10 (2000) 020 [hep-th/0009182] [INSPIRE].

[7] E. Bergshoeff, J. Gomis and Z. Yan, Nonrelativistic String Theory and T-duality, JHEP 11 (2018) 133 [arXiv: 1806.06071] [INSPIRE].

[8] J. Gomis, J. Gomis and K. Kamimura, Non-relativistic superstrings: A New soluble sector of $A d S_{5} \times S^{5}, J H E P 12$ (2005) 024 [hep-th/0507036] [INSPIRE].

[9] J. Brugues, T. Curtright, J. Gomis and L. Mezincescu, Non-relativistic strings and branes as non-linear realizations of Galilei groups, Phys. Lett. B 594 (2004) 227 [hep-th/0404175] [INSPIRE].

[10] R. Andringa, E. Bergshoeff, J. Gomis and M. de Roo, 'Stringy' Newton-Cartan Gravity, Class. Quant. Grav. 29 (2012) 235020 [arXiv:1206.5176] [InSPIRE].

[11] J. Brugues, J. Gomis and K. Kamimura, Newton-Hooke algebras, non-relativistic branes and generalized pp-wave metrics, Phys. Rev. D 73 (2006) 085011 [hep-th/0603023] [INSPIRE].

[12] E.A. Bergshoeff, J. Gomis, J. Rosseel, C. Şimşek and Z. Yan, String Theory and String Newton-Cartan Geometry, J. Phys. A 53 (2020) 014001 [arXiv:1907.10668] [INSPIRE].

[13] T. Harmark, J. Hartong, L. Menculini, N.A. Obers and G. Oling, Relating non-relativistic string theories, JHEP 11 (2019) 071 [arXiv: 1907.01663] [INSPIRE].

[14] J. Gomis, J. Oh and Z. Yan, Nonrelativistic String Theory in Background Fields, JHEP 10 (2019) 101 [arXiv: 1905.07315] [INSPIRE].

[15] U.H. Danielsson, A. Guijosa and M. Kruczenski, Newtonian gravitons and D-brane collective coordinates in wound string theory, JHEP 03 (2001) 041 [hep-th/0012183] [INSPIRE].

[16] N. Seiberg, L. Susskind and N. Toumbas, Strings in background electric field, space/time noncommutativity and a new noncritical string theory, JHEP 06 (2000) 021 [hep-th/0005040] [INSPIRE].

[17] R. Gopakumar, J.M. Maldacena, S. Minwalla and A. Strominger, S duality and noncommutative gauge theory, JHEP 06 (2000) 036 [hep-th/0005048] [INSPIRE].

[18] E.S. Santos, M. de Montigny, F.C. Khanna and A.E. Santana, Galilean covariant Lagrangian models, J. Phys. A 37 (2004) 9771 [InSPIRE].

[19] E. Bergshoeff, J. Rosseel and T. Zojer, Non-relativistic fields from arbitrary contracting backgrounds, Class. Quant. Grav. 33 (2016) 175010 [arXiv: 1512.06064] [INSPIRE]. 
[20] G. Festuccia, D. Hansen, J. Hartong and N.A. Obers, Symmetries and Couplings of Non-Relativistic Electrodynamics, JHEP 11 (2016) 037 [arXiv:1607.01753] [INSPIRE].

[21] C. Batlle, J. Gomis and D. Not, Extended Galilean symmetries of non-relativistic strings, JHEP 02 (2017) 049 [arXiv:1611.00026] [INSPIRE].

[22] J. Polchinski, String theory. Vol. 1: An introduction to the bosonic string, Cambridge Monographs on Mathematical Physics, Cambridge University Press (2007) [DOI] [INSPIRE].

[23] A. Bagchi, R. Basu, A. Kakkar and A. Mehra, Galilean Yang-Mills Theory, JHEP 04 (2016) 051 [arXiv: 1512.08375] [INSPIRE].

[24] C. Hull and B. Zwiebach, Double Field Theory, JHEP 09 (2009) 099 [arXiv:0904.4664] [INSPIRE].

[25] J. Gomis, Z. Yan and M. Yu, T-duality in Nonrelativistic Open String Theory, JHEP 02 (2021) 087 [arXiv:2008.05493] [inSPIRE].

[26] T. Harmark, J. Hartong, L. Menculini, N.A. Obers and Z. Yan, Strings with Non-Relativistic Conformal Symmetry and Limits of the AdS/CFT Correspondence, JHEP 11 (2018) 190 [arXiv: 1810.05560] [INSPIRE].

[27] Z. Yan and M. Yu, Background Field Method for Nonlinear Sigma Models in Nonrelativistic String Theory, JHEP 03 (2020) 181 [arXiv:1912.03181] [INSPIRE].

[28] A.D. Gallegos, U. Gürsoy and N. Zinnato, Torsional Newton Cartan gravity from non-relativistic strings, JHEP 09 (2020) 172 [arXiv: 1906.01607] [INSPIRE].

[29] W. Kummer and D.V. Vassilevich, Renormalizability of the open string sigma model and emergence of D-branes, JHEP 07 (2000) 012 [hep-th/0006108] [INSPIRE].

[30] A. Abouelsaood, C.G. Callan Jr., C.R. Nappi and S.A. Yost, Open Strings in Background Gauge Fields, Nucl. Phys. B 280 (1987) 599 [inSPIRE].

[31] D. Roychowdhury, Probing tachyon kinks in Newton-Cartan background, Phys. Lett. B 795 (2019) 225 [arXiv: 1903.05890] [INSPIRE].

[32] D. Pereñiguez, p-brane Newton-Cartan geometry, J. Math. Phys. 60 (2019) 112501 [arXiv: 1908.04801] [INSPIRE].

[33] J. Klusoň, Non-Relativistic D-brane from T-duality Along Null Direction, JHEP 10 (2019) 153 [arXiv: 1907.05662] [INSPIRE].

[34] J. Klusoň, Unstable D-brane in Torsional Newton-Cartan Background, JHEP 09 (2020) 191 [arXiv: 2001.11543] [INSPIRE].

[35] T. Harmark, J. Hartong and N.A. Obers, Nonrelativistic strings and limits of the AdS/CFT correspondence, Phys. Rev. D 96 (2017) 086019 [arXiv: 1705.03535] [INSPIRE].

[36] K. Banerjee, R. Basu and A. Mohan, Uniqueness of Galilean Conformal Electrodynamics and its Dynamical Structure, JHEP 11 (2019) 041 [arXiv: 1909.11993] [INSPIRE].

[37] S. Chapman, L. Di, K.T. Grosvenor and Z. Yan, Renormalization of Galilean Electrodynamics, JHEP 10 (2020) 195 [arXiv:2007.03033] [INSPIRE]. 